\title{
Collapse of void arrays under stress wave loading
}

\author{
A. B. SWANTEK AND J. M. AUSTINं \\ Department of Aerospace Engineering, University of Illinois, Urbana, IL 61801, USA
}

(Received 6 April 2009; revised 17 November 2009; accepted 17 November 2009)

The interaction of an array of voids collapsing after passage of a stress wave is studied as a model problem relevant to porous materials, for example, to energy localization leading to hotspot formation in energetic materials. Dynamic experiments are designed to illuminate the hydrodynamic processes of collapsing void interactions for eventual input into device-scale initiation models. We examine a stress wave loading representative of accidental mechanical insult, for which the wave passage length scale is comparable with the void and inter-void length scales. A single void, two-void linear array, and a four-void staggered array are studied. Diagnostic techniques include high-speed imaging of cylindrical void collapse and the first particle image velocimetry measurements in the surrounding material. Voids exhibit an asymmetrical collapse process, with the formation of a high-speed internal jet. Volume and diameter versus time data for single void collapse under stress wave loading are compared with literature results for single voids under shock-wave loading. The internal volume history does not fall on a straight line and is in agreement with simulations, but in contrast to existing linear experimental data fits. The velocity field induced in the surrounding material is measured to quantify a region of influence at selected stages of single void collapse. In the case of multiple voids, the stress wave diffracts in response to the presence of the upstream void, affecting the loading condition on the downstream voids. Both collapse-inhibiting (shielding) and collapsetriggering effects are observed.

\section{Introduction}

The formation of regions of localized energy release, or hotspots, is critical to detonation initiation in energetic materials. Hotspots can result in local ignition kernels at conditions in which bulk chemical energy release is insufficient for initiation. Hotspots may be formed due to the interaction of the loading wave with micro-scale and molecular-scale material heterogeneities through processes such as debonding, micro-cracking and shear-banding (Bowden \& Yoffe 1952). One mechanism by which hotspots can be created is the collapse of voids. If the chemical and mechanical energy release can couple and overcome dissipation, a detonation is initiated (Tarver, Chidester \& Nichols 1996). Understanding the dominant mechanisms of hotspot formation is critical for determining ignition performance, as well as establishing safe handling procedures.

Predicting hotspot criticality, ignition spread and detonation initiation is an extremely challenging problem, involving thermo-mechanical fluid-structure coupling and complex chemical kinetics. In addition, the relevant processes span an extremely 
broad range of length and time scales. At the device scale, the goal is usually to predict whether a detonation is initiated and the run to detonation distance; however, hotspot formation occurs at the substantially smaller scales associated with the material structure. A considerable challenge must therefore be addressed: How can predictions performed on the device scale incorporate the critical impact of phenomena at unresolved scales? Applications such as shock desensitization (Campbell \& Travis 1985) and the formation of dead zones (Ferm et al. 2001) are particularly relevant as they are not easily treated by ignition and growth (Tarver, Hallquist \& Erickson 1985) or Johnson-Tang-Forest (JTF) models (Johnson, Tang \& Forest 1985).

Scale-bridging codes are intended to span between device and material structure length scales (Menikoff 2001; Baer 2002; Nichols \& Tarver 2002). In the statistical modelling approach, identical and individual hotspots are presumed to occur with the same statistical distribution as the voids in the unreacted material. However, the interaction of loading wave with the upstream voids has been shown to have an impact on the collapse of downstream voids (Dear \& Field 1988; Bourne \& Field 1990). The overarching goal of this work is to examine the interactive collapse of a void array to contribute to model development for scale-bridging codes.

Numerous researchers (e.g. Mader 1965; Carroll \& Holt 1972; Khasainov et al. 1981; Maiden \& Nutt 1986; Kang, Butler \& Baer 1992; Bourne \& Field 1999; Menikoff 2003b; Tran \& Udaykumar 2006) have examined energy localization mechanisms during the collapse of a single void, identifying several relevant mechanisms: shock focusing, adiabatic gas compression, jetting, hydrodynamic and viscoplastic work. A Reynolds number $R e$ based on the void size can be defined as

$$
\operatorname{Re}=\delta_{o} \sqrt{\rho p_{s}} / \mu
$$

where $\delta_{o}$ is the critical void size, $p_{s}$ is the loading wave strength, and $\rho$ and $\mu$ are the density and viscosity, respectively, in the surrounding media (Khasainov et al. 1981). For small Reynolds numbers corresponding to small void diameters, the collapse is in a viscous regime. For high Reynolds numbers and larger voids, the collapse is in the hydrodynamic regime (Khasainov et al. 1981; Tran \& Udaykumar 2006). In this study, we focus on the hydrodynamic processes that occur as an array of voids interact during the collapse. We note that the experiments are designed to examine a model problem in a gelatinous material and do not reproduce the material properties of explosives.

There is an extensive literature examining the collapse of voids or bubbles under shock loading which, for energetic materials, is applicable in performance (highimpact velocity) applications. In this work, we instead examine a slower loading wave with a ramped profile, typical of an accidental (low-impact velocity) scenario in which a stress wave is induced in the device casing. The length scales of the loading wave profile are comparable with the void and inter-void length scales, potentially resulting in a strongly coupled interaction. Note that the relative importance of void collapse in hotspot formation depends on the type of explosive. In crystalline primary explosives, lower impact loading can increase the relative importance of mechanisms such as crystalline fracture and shear-banding. In secondary explosives such as PETN, void collapse plays a more substantial role. The inclusion of bubbles in liquid, gelatinous or plastic explosives render it sensitive to the 'gentlest' impact (Bowden \& Yoffe 1952). Stress wave loading of voids also occurs in numerous other applications from hydraulic to biomedical. In shock-wave lithotripsy, for example, the interaction 
between shearing and cavitation can cause substantial damage to surrounding tissue material (Bailey et al. 2003).

We carry out dynamic experiments in a model set-up similar to that pioneered by Dear et al. (1988) for a different loading condition. Gas-filled voids in a thin sheet of gelatinous material are sandwiched between two optically transparent plates and subjected to a planar stress wave induced by a gas gun and striker plate. High-speed images are used to track the precursor wave and void interfaces as a function of time. In addition, full velocity field data during passage of the loading wave and collapse of the void array are obtained via particle image velocimetry (PIV) measurements.

The paper is organized as follows. In $\S 2$, we summarize relevant literature studies of shock-induced void collapse, for comparison with the current stress wave loading results. The experimental set-up and diagnostics are described in $\S 3$, including the characterization of the loading wave. Results including high-speed movies tracking the void array collapse and velocity field data in the surrounding media follow. We first discuss a single void in $\S 4$, examining the key processes occurring during collapse and quantify the region of influence. A longitudinal two-void array $(\S 5)$ and a fourvoid staggered array $(\S 6)$ are then examined, with a focus on the dynamics of void interaction with each other and with the loading wave during collapse. In $\S 7$, results of this study under conditions of stress wave loading are compared and contrasted with results for bubble collapse under shock-wave loading.

\section{Previous shock-induced void collapse research}

In addition to their critical role in the condensed phase detonation initiation, shock-cavity interactions occur in a wide range of applications. Extensive work, both experimental and computational, has been performed for the collapse of a single void in reactive and non-reactive media under shock loading. Ding \& Gracewski (1996) performed a two-dimensional computational study of a gas cavity exposed to shocks of various strengths. A weak shock was defined as one that resulted in symmetric cavity collapse, which was observed to occur if the pressure ratio across the shock wave was less than 300. A strong shock was defined as one that resulted in asymmetric cavity collapse, which was observed to occur if the pressure ratio was greater than 5000. Intermediate cases were not investigated in this study. In the case of a 'light' bubble (with the acoustic impedance of the material inside the cavity less than that of the surrounding material), a reflected expansion wave is generated upon the shock interaction with the void interface. The expansion wave was observed to induce a particle velocity nearly twice that of the shock-induced velocity. For the strong shock case, the upstream interface rapidly coalesces into a jet, which impinges upon the distal interface, asymmetrically collapsing the cavity.

The formation of an internal, high-speed jet was experimentally observed by Dear et al. (1988) and Bourne \& Field (1992), in the study of a 0.26 GPa shock interaction with $3-12 \mathrm{~mm}$ diameter cylindrical voids in a $12 \%$ gelatine--water mixture. The jet tip was observed to propagate at roughly twice the post-shock particle velocity, which is in agreement with the acoustic approximation. Additionally, the authors estimate that the jet velocity could increase another factor of 1.5 due to nonlinear effects. A summary of normalized volume versus time data was presented and compared with existing results from the literature. Bourne \& Field (1992) found a linear curve fit to these data. Strong shocks with velocities corresponding to pressure ratios up to $3.49 \mathrm{GPa}$ were also investigated in a related study (Bourne \& Field 1990). In this case, the internal jet was observed to propagate through the void ahead of the incident 
shock with a velocity of 7.5 times that of the particle velocity in the medium. The impact of the jet on the downstream wall created a shock wave that overtook the incident loading wave.

The internal, high-speed jet that may be formed during the shock-bubble interaction and the consequent 'water-hammer' impact on the material downstream of the void has been examined numerically and experimentally by numerous researchers. For example, in an inert material with constitutive properties of HMX, Menikoff $(2003 b)$ observed that under strong shock loading, the internal jet propagated at velocities comparable with the shock velocity, and its impact on the distal interface generated a Mach reflection in the lead shock. Ball et al. (2000) simulated a cylindrical, $6 \mathrm{~mm}$ diameter, air-filled void in water for comparison with the experiments of Bourne \& Field (1992). When exposed to a $1.9 \mathrm{GPa}$ shock, the void was found to collapse asymmetrically and form a high-speed jet. The impact of this jet on the distal wall created a $4.7 \mathrm{GPa}$ blast wave that propagated into the downstream media. Bourne \& Milne (2003) discuss the importance of the jets in the ignition process. Experimental and numerical results indicate that a reaction zone is always formed ahead of the lead shock. When comparing simulations, they saw that in the reactive case, the shock is accelerated in comparison with the inert case, illustrating the importance of coupling between the fluid dynamics and the chemical energy release.

Bourne \& Field (1991) also examined the initiation of reaction through void collapse by sensitizing a $12 \%$ gelatine-water mixture with an ammonium nitrate/sodium nitrate emulsion. The high-speed Schlieren revealed that during the collapse, a series of shocks were reflected internally inside the void, resulting in gas heating. During the final stages of the collapse, luminescence was observed indicating high temperatures inside the cavity. By measuring the volume of the gas inside the void, Dear et al. (1988) estimate that for an adiabatic compression temperatures would reach $750 \mathrm{~K}$, at a minimum. This result is also consistent with gas luminescence due to both free-radical creation and radiative recombination. Spectroscopic measurements made by Tarver et al. (1996) confirmed this; temperatures in the range of 600-1600 K were obtained. These results illustrate that ignition temperatures can be reached at the final stages of void collapse.

Ball et al. (2000) and Turangan et al. (2008) report the normalized volume versus time behaviour observed in their simulations of cylindrical cavity collapse. Their data exhibit a non-constant rate of collapse and deviates from the linear experimental data fit of Bourne \& Field (1992). Johnsen \& Colonius (2009) simulated the collapse of a spherical air bubble in water, examining shock-induced collapse and Rayleigh collapse in a free field and near a rigid boundary. A range of pressure ratios were examined. Normalized volume versus time data show a collapse history that does not fall on a straight line. We compare our experimental results for the baseline case of a single void collapse with these experimental and numerical studies, to quantify the effects of stress wave versus shock-wave loading.

In addition to their work on isolated cavities, Dear \& Field (1988) have examined the collapse of an array of voids under shock loading. The study considered a one by three longitudinal array of $3 \mathrm{~mm}$ voids at $5 \mathrm{~mm}$ spacing. The downstream voids were observed to experience only a slight compression due to shock passage. The collapse of the second cavity is a result of the 'rebound shock' generated by the upstream void. Similar observations were made by Dear et al. (1988), who concluded that for correct conditions of shock strength, cavity diameter and cavity spacing, a chain reaction of collapses is attainable. Vertical three by one arrays were also investigated. 
Side void jets were found to diverge from their centrelines towards the central void. Lastly, the study involved the examination of a three by three rectangular array of cavities. The outside cavities produced slightly divergent jets as in the vertical array case. In addition, the first column of cavities shielded subsequent columns, as in the longitudinal case. The collapse of downstream columns was observed to be a result of the rebound shock from the upstream column.

In the above studies, the loading condition is a shock, such as would be induced by high-velocity projectile impact. In this study, we instead examine a stress wave loading condition that, in an energetic material, might be induced by accidental mechanical insult. Stress wave loading is also of interest in other applications, for example in biomedicine. We choose the length scales of the stress loading wave profile to be comparable with the void and inter-void length scales, and examine the interaction of the loading wave and dynamics of cavity collapse. The final pressure ratio across the loading wave in this study is intermediate to the weak and strong cases identified by Ding \& Gracewski (1996), and asymmetric collapse is observed for an isolated void. A single void, two-void linear array, a four-void staggered array are studied. Diagnostic techniques include high-speed imaging of void collapse and PIV measurements in the surrounding media. In the case of a single void, we compare and contrast our results with those of the existing shock-induced void collapse literature.

\section{Experimental set-up}

The gas gun used in this experiment has a barrel length of $2.1 \mathrm{~m}$ and a honed $25 \mathrm{~mm}$ inner diameter. A projectile, made from heat treated and hardened maraging steel, is accelerated from a reservoir with an internal piston pressurized with compressed air. Near the end of the gun barrel, two sets of infrared emitters and detectors are positioned to measure the projectile velocity. In these experiments, the reservoir is pressurized to $15 \mathrm{psi}$ and the resulting projectile velocity is $27.0 \pm 0.3 \mathrm{~m} \mathrm{~s}^{-1}$.

Void collapse in energetic materials involves complex interaction between thermochemical, mechanical, and hydrodynamic processes. In this study, we examine hydrodynamic mechanisms in a model problem. The test sample is made by sandwiching a thin sheet of gelatinous material between two optically accessible plates. A mixture of agarose (GenePure LE Agarose, supplied by ISC BioExpress) and glycerol gradient buffer (GGB) is set as a thin $(1.6 \mathrm{~mm})$ gelatinous sheet into which cylindrical, air-filled voids can be easily introduced. The gel is manufactured by dissolving $5 \%$ agarose by volume in GGB at $295 \mathrm{~K}$. The mixture is heated until the boiling point is reached, cooled to $328 \mathrm{~K}$ and poured into a mold containing $3 \mathrm{~mm}$ cylindrical tubing used to form the voids. This 'guide plate' produces highly repeatable void geometries with distinct interfaces. Different guide plates are used to create different array geometries. After the gel has set, the guide plate is removed and replaced by a solid side plate. A comparable gel material (polydimethylsiloxane, PDMS) was used in studies of dynamic witness plates for exploding bridge wire detonators (Murphy et al. 2005; Murphy \& Adrian 2007). Similar experimental techniques were used as in the present study, with the transparent gel material allowing optical access for Schlieren visualization of the blast wave and PIV measurements in the post-shock fluid. The mold, shown in figure 1 , is made from two $100 \mathrm{~mm} \times$ $70 \mathrm{~mm} \times 9.5 \mathrm{~mm}$ (length $\times$ width $\times$ height) pieces of polymethyl methacrylate (PMMA) serving as windows and two spacers of dimension $100 \mathrm{~mm} \times 20 \mathrm{~mm} \times$ $1.6 \mathrm{~mm}$. The sample thickness $(1.6 \mathrm{~mm})$ is chosen to minimize three-dimensional effects. 


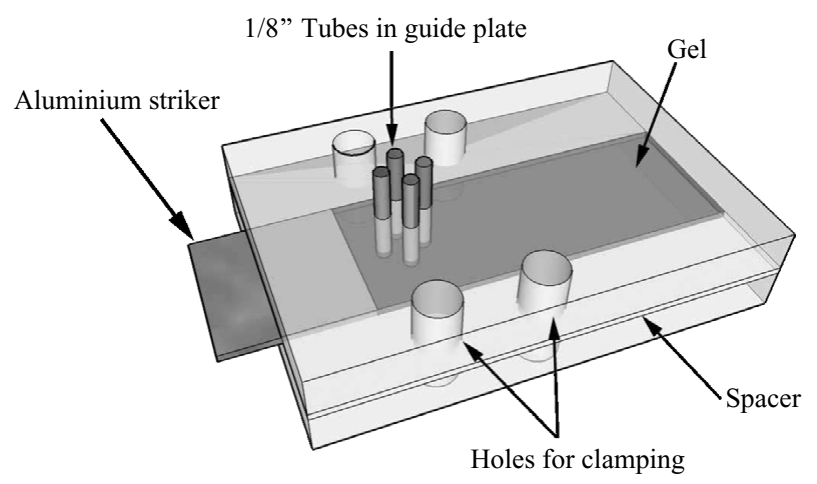

FIGURE 1. Sketch of mold used to create samples of gelatinous material with voids. The guide plate is replaced after the gel has set. The projectile impacts the striker plate that transmits the loading waves to the sample.

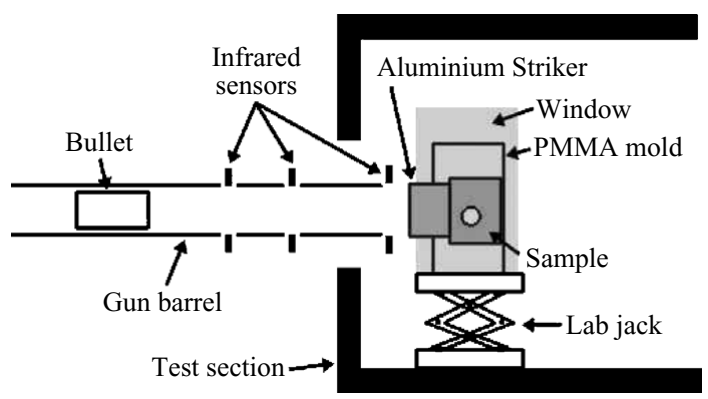

FIGURE 2. Schematic of gas gun and test section showing the projectile, projectile velocity measurement and diagnostic trigger sensors, and sample location.

The sample state in the mold may be approximated as plane strain. An aluminium striker plate is used to create the loading condition of interest. The projectile impacts the striker plate, introducing a stress wave that produces the loading condition in the gel material. The striker has a total length of $30 \mathrm{~mm}$ and protrudes $15 \mathrm{~mm}$ into the mold, so that the usable gel test sample is $85 \mathrm{~mm} \times 30 \mathrm{~mm} \times 1.6 \mathrm{~mm}$. The spacing between the striker plate and the centre of the first (upstream) void is $5 \mathrm{~mm}$. The four pieces are sealed with Dow Corning 732 RTV Multipurpose Sealant and clamped together.

The material properties of agarose-GGB gel were obtained from a separate series of experiments. In order to determine the Young's modulus, a quasi-static compression test using an MTS Alliance RT/30 load frame was conducted. The test was performed on seven samples, yielding an average Young's modulus value of $38.2 \pm 4.0 \mathrm{kPa}$. This matched well with results obtained for gelatine by Kodama \& Tomita (2000). The sound speed of the Agarose-GGB gel was obtained using a JSR Ultrasonics PR35 Ultrasonic Pulser/Receiver. This resulted in an average sound speed of $1500 \pm 10 \mathrm{~m} \mathrm{~s}^{-1}$. As we consider a model problem, the material properties of the gelatine differ significantly from a solid explosive.

Inside the test section, the sample is placed without restraint immediately downstream of the exit of the barrel. The experimental diagnostics trigger sensor consists of an infrared emitter and sensor pair and is mounted $5 \mathrm{~mm}$ downstream of the barrel exit. A schematic of the gas gun and test section is shown in figure 2 . 


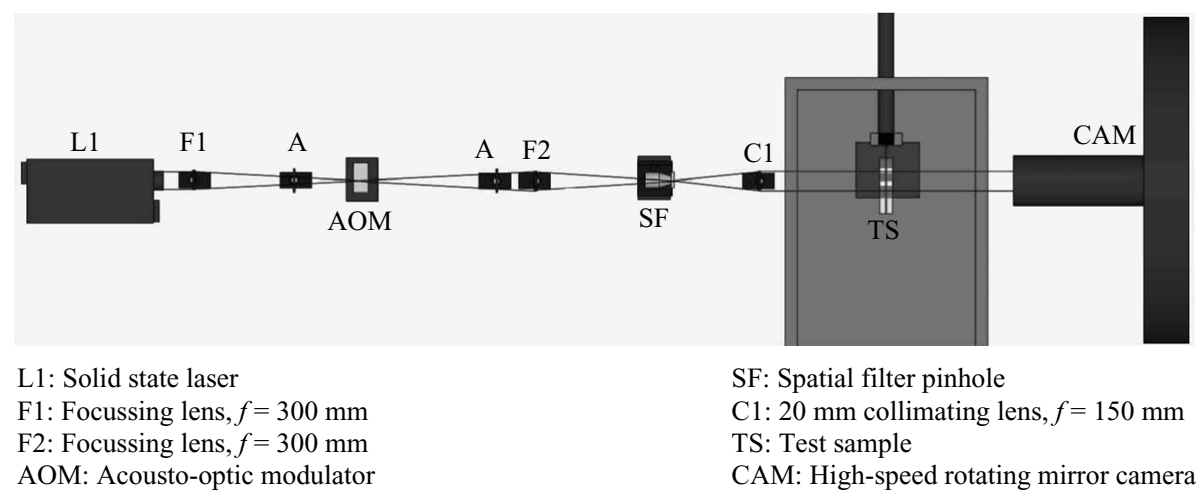

A: Aperture

FIGURE 3. Schematic of the high-speed imaging set-up.

A Utah Image Systems' high-speed, rotating mirror framing camera is used to acquire time-resolved images of the void collapse process. A 2 Watt $532 \mathrm{~nm}$ solid state continuous wave laser provided illumination for the experiment. The $200 \mathrm{~ns}$ exposure time is controlled by an acousto-optic modulator. The light leaving the acousto-optic modulator passes through a $10 \mu \mathrm{m}$ pinhole, creating a point source, which is then collimated $(f=150 \mathrm{~mm})$ to a $20 \mathrm{~mm}$ field of view. The camera produces a total of 80 images on two separate film tracks inside the circumference of the camera drum. A schematic of the optical configuration is shown in figure 3 . The interframe time can be varied and is set to either $1.5 \mu$ s or $2.0 \mu$ s depending upon the void configuration. Error bars on high-speed image data are $\pm 0.23 \mathrm{~mm}$ in position and $\pm 0.75 \mu \mathrm{s}$ and $\pm 1.0 \mu \mathrm{s}$ in time for the $1.5 \mu \mathrm{s}$ and $2.0 \mu \mathrm{s}$ interframe times, respectively. These images not only serve to track the void interfaces with time but can provide void area (and subsequently volume assuming a constant sample thickness of $1.6 \mathrm{~mm}$ ) as a function of time during the collapse. A MATLAB code, including edge-detection algorithms, has been implemented to track the boundary of the void and compute the enclosed area for each film frame.

PIV measurements are conducted using a two-colour, single-frame technique. Hollow glass spheres, approximately $20 \mu \mathrm{m}$ in diameter, are introduced into the gel before it is poured into the mold. The PIV set-up (figure 4) consists of a pair of fast response, high-energy LEDs by Innovative Scientific Solutions Inc. (ISSI). After receiving a triggering signal from the infrared sensors, a delay generator triggers the first LED, which emits a $500 \mathrm{~ns}$ red $(625 \mathrm{~nm})$ light pulse. After a $3 \mu$ s delay, a second LED emits a $500 \mathrm{~ns}$ blue $(625 \mathrm{~nm})$ light pulse. Light from the two LEDs passes through a prism and illuminates a $30 \mathrm{~mm}$ field of view in the gel. A Nikon D50 camera is triggered manually. The raw data are analysed using dPIV 2.1 (ISSI) software. The resolution of the velocity measurements is $2 \mathrm{~m} \mathrm{~s}^{-1}$.

\subsection{Characterizing the stress wave loading condition}

In performance applications, a strong shock wave is used to initiate detonation in energetic materials. There has been a significant amount of research examining the related problem of shock-induced collapse of bubbles. The type of collapse can be identified as symmetric or asymmetric, parameterized by the pressure ratio across the shock wave. For the larger bubbles or voids that are relevant to the hydrodynamic collapse regime, the shock passage time is typically much less than the void collapse time (Bourne \& Field 1992). 


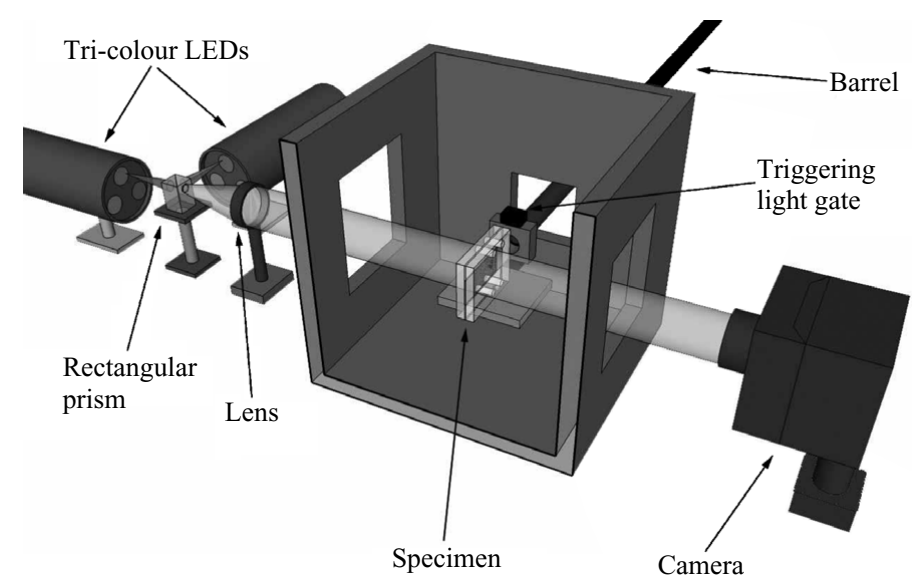

FIGURE 4. Schematic of the two-colour, single-frame PIV experiment.

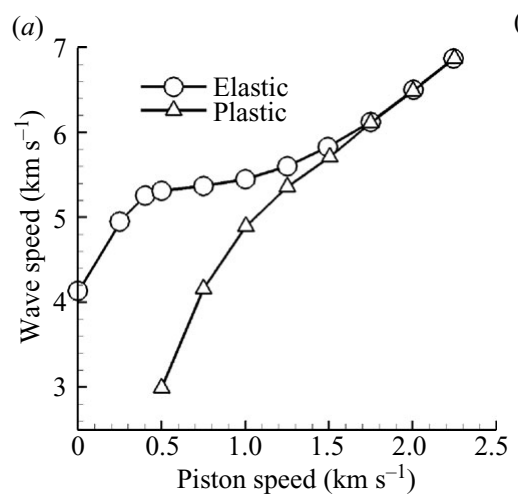

(b)

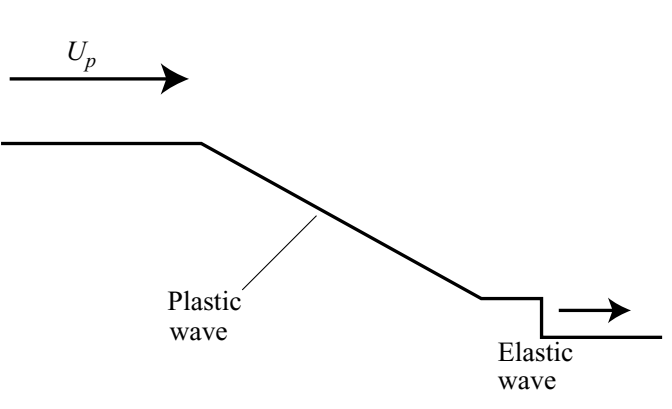

FIGURE 5. (a) Wave velocity vs. piston velocity in HMX from Jaramillo et al. (2007). The present experiments examine a lower impact velocity that produces an elastic-plastic wave in the striker plate. (b) Representation of an elastic-plastic wave.

In this work, we instead consider a lower-velocity loading condition, such as one that might result from accidental impact. For example, Jaramillo, Sewell \& Strachan (2007, see figure 5a), Menikoff (2003a) and Dick et al. (2004) report on the development of the elastic-plastic wave propagation in HMX as a function of impact velocity. At low piston speeds, only a precursor wave is present. At intermediate piston speeds, an elastic-plastic stress wave propagates through the material. Finally, at the highest piston speeds, a shock wave is formed. We examine a loading condition that is in the intermediate, stress wave regime. In this experiment, a projectile with velocity of $27 \mathrm{~m} \mathrm{~s}^{-1}$ impacts a striker plate and produces an elastic-plastic wave in the plate. The resulting loading condition in the gel (figure $5 b$ ) consists of a pressure pulse generated by the incidence of the precursor elastic wave, followed by a ramped velocity profile generated by the incidence of the plastic wave. Through the ramped wave, the particle velocity in the gel sample increases to match the projectile velocity. The length scales of the ramped velocity wave profile are comparable to the void diameter and intervoid spacing, potentially resulting in a dynamic interaction between the propagating loading wave and the collapsing voids. 


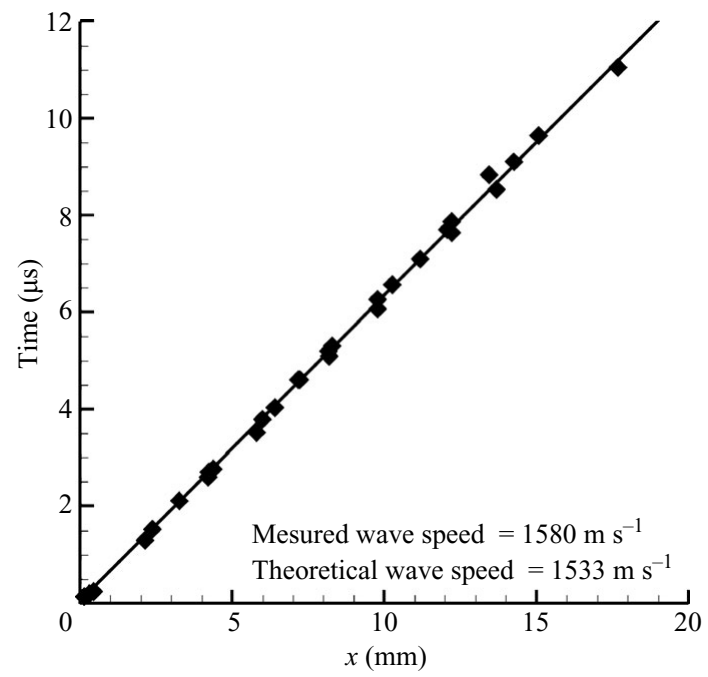

FIGURE 6. High-speed image $x-t$ data of pressure pulse resulting from the elastic wave incident on the striker-sample interface.
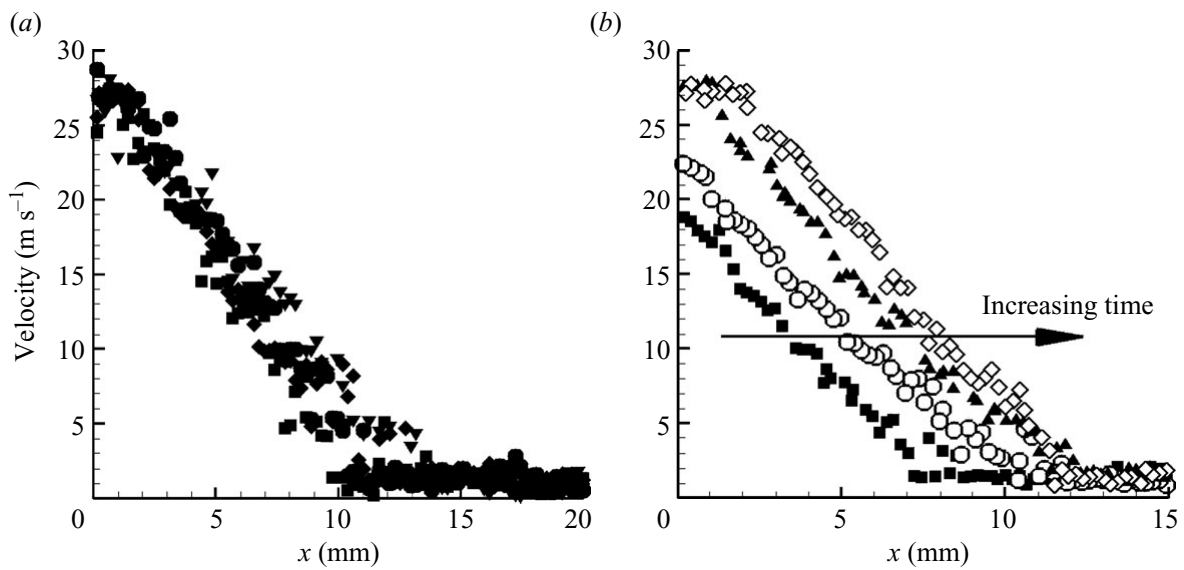

FIGURE 7. (a) Velocity profiles through the Agarose-GGB mixture measured at several cross-sections in a single PIV image and $(b)$ evolution of centreline velocity as the stress wave propagates through the field of view. The striker plate is located at $x=0 \mathrm{~mm}$. Flow is from left to right.

We first quantify the loading condition in the gel material without voids. The pressure pulse resulting from the elastic precursor wave is tracked using high-speed images. These data are shown on an $x-t$ diagram in figure 6 . The pulse is measured to propagate at $1580 \pm 38 \mathrm{~m} \mathrm{~s}^{-1}$. From the PIV measurements, we observe that the precursor wave induces no detectable velocity field (figure 7).

The incidence of the plastic wave on the striker-gel material interface results in a wave with a ramped linear velocity profile propagating through the gel material. The ramped velocity profile contrasts the discontinuous velocity profile that results from shock-wave loading. To characterize the loading condition, we measure velocity profiles at several horizontal cross-sections in the gel from a single PIV image (figure $7 a$ ). These data are obtained $17 \mu$ s after projectile impact on the striker, 


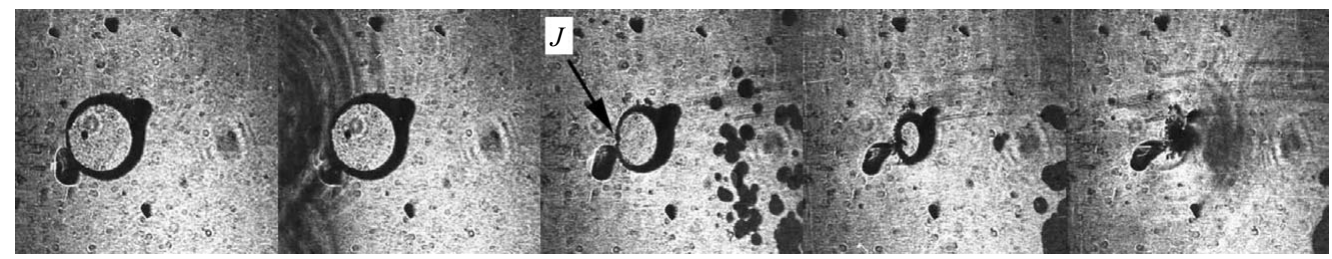

(a) $0 \mu \mathrm{s}$

(b) $4.5 \mu \mathrm{s}$

(c) $18 \mu \mathrm{s}$

(d) $25.5 \mu \mathrm{s}$

(e) $33 \mu \mathrm{s}$

FIGURE 8. High-speed images of a single void collapse. Images are obtained using a laser light source through a gelantinous material; some laser diffraction could not be eliminated. Initial void diameter is $3 \mathrm{~mm}$. In $(c), J$ indicates the genesis of the jet.

approximately $11 \mu \mathrm{s}$ after the precursor enters the gel. The velocity profile increases monotonically to the projectile velocity. These data are compared to establish that the wavefront is reasonably planar over approximately $25 \mathrm{~mm}$, with similar velocity profiles at each cross-section examined. The evolution of the stress wave as it propagates through the experimental field of view is shown in figure $7(b)$. Data have been translated so that the striker interface is always located along the $y$ axis. The first two profiles show the stress wave entering the sample; the final two profiles show the fully developed velocity field increasing approximately linearly up to the striker velocity of $27 \mathrm{~m} \mathrm{~s}^{-1}$. These PIV images obtained at different times during the wave propagation through the sample show that no wave attenuation was evident in the absence of voids. No loading wave or velocity field non-uniformity because of non-ideal material properties was observed in these or other PIV images.

\section{Collapse of a single void}

We first examine the baseline case of a single void using high-speed images to obtain time-resolved visualization of the collapse. These images also subsequently serve as a time reference in the analysis of the single-shot PIV images. Figure 8 shows the collapse of a cylindrical void exposed to a stress wave. We calculate the ratio of post-wave pressure to the initial pressure $p / p_{o}$ to be 421 , which is intermediate to the weak $\left(p / p_{o}<300\right)$ and strong $\left(p / p_{o}>5000\right)$ regimes defined for the shock-induced collapse (Ding \& Gracewski 1996). Loading conditions between these two cases were not studied, and to the authors' knowledge, the boundary between the two regimes has not been established. Qualitatively, we observe that the void undergoes an asymmetric collapse as in the strong shock regime, with a high-speed jet evident in the images. The times shown are the elapsed times referenced from figure $8(a)$. The asymmetric collapse is due to the initial arrival of the loading wave on the proximal void interface while the distal interface is unaffected and remains stationary.

The arrival of the precursor pulse at the upstream interface of the void is seen in figure $8(b)$. Significant wavefront curvature can be observed; however, this is assumed to have negligible effect, as the precursor pulse does not trigger the void collapse. Instead, as will be seen in the PIV data, the void begins to collapse with the arrival of the stress wave. The genesis of the jet is visible in figure $8(\mathrm{c})$, becoming fully developed in figure $8(d)$. Figure $8(e)$ shows the consequence of the jet impingement on the distal interface of the void. A forward-facing, semi-circular pulse propagates into the downstream material. The time to minimum diameter (i.e. collapse time based upon the jet reaching the downstream interface) $t_{D, \min }$ is found to be $24 \pm 1.5 \mu \mathrm{s}$. Note 

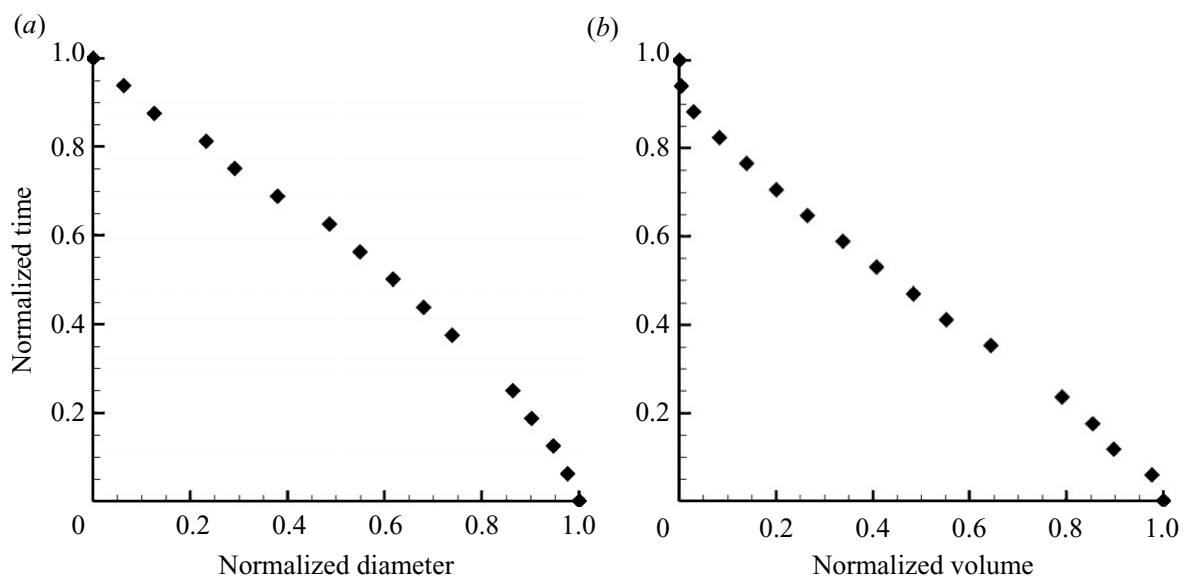

FIGURE 9. Normalized $(a)$ centreline diameter and $(b)$ internal volume vs. time for a single void collapse.

that this is not the same as the time to reach minimum volume $t_{V, \min }$ for the cases where a jet appears in the collapse. For the single void, $t_{V, \min }$ is $25.5 \pm 1.5 \mu \mathrm{s}$.

The normalized cavity centreline cross-sectional diameter versus time and the normalized cavity internal volume versus time based on cavity interface location are reported in figures $9(a)$ and $9(b)$, respectively. The centreline diameter versus time plot shows an initial period of acceleration up to $t / t_{D, \min } \approx 0.25$. The downstream interface is stationary while the upstream accelerates. The subsequent region appears linear until the jet reaches the distal interface. The cavity volume versus time exhibits similar behaviour at the beginning of the collapse. Again, acceleration is observed in the beginning stages; however, this portion extends only up to $t / t_{V, \min } \approx 0.12$. Towards the end of the collapse, starting at $t / t_{V, \min } \approx 0.82$, a deceleration of the volume versus time is observed, potentially because of the increased gas compression within the void. Diameter and volume histories are compared and contrasted with literature results in $\S 7$.

Single-shot, two-colour, single-frame PIV measurements are made at selected times after projectile impact. Figure 10 shows the planar velocity field at three stages during the single void collapse process. The grey boundary represents the perimeter of the void during the first PIV frame, and the red boundary represents the perimeter of the void during the second PIV frame. The black dotted boundary represents the initial size and location of the void, and the white fill region represents the uncollapsed area of the void after the second frame. During the early stages of the collapse, there is a region upstream of the void where the velocity is significantly greater than that in the free stream (figure 10a). The velocity approaches more than three times the post-wave particle velocity. The stress wavefront diffracts around the void, and corresponding velocity vector field divergence above and below the void, with a shielded region of zero velocity just downstream of the void, is observed in the PIV images (figures $10 a$ and $10 b$ ). In the final image obtained during the cavity collapse (figure 10c), the consequence of the wave generated by the jet impingement on the distal void interface observed in high-speed image data is presented. Radial velocity vectors appear in the previously shielded downstream region of the void, with a profile similar to that of the wave seen in figure 8(e). The wave appears to advance ahead of the initial stress wave propagating through the gel. 
(a)

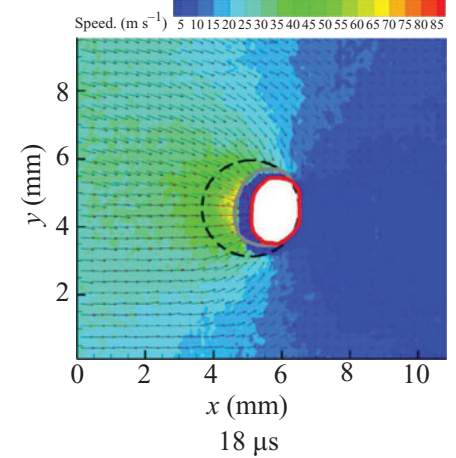

(b)

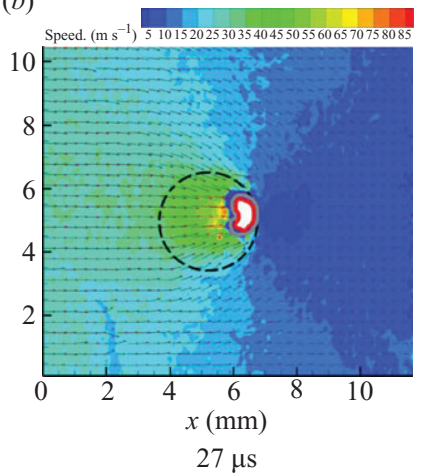

(c)

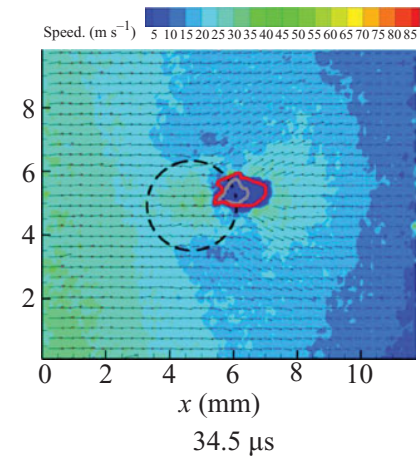

FIGURE 10. Velocity contours for a single void collapse. Times are referenced from the same scale as in figure 8 . Initial void diameter is $3 \mathrm{~mm}$.
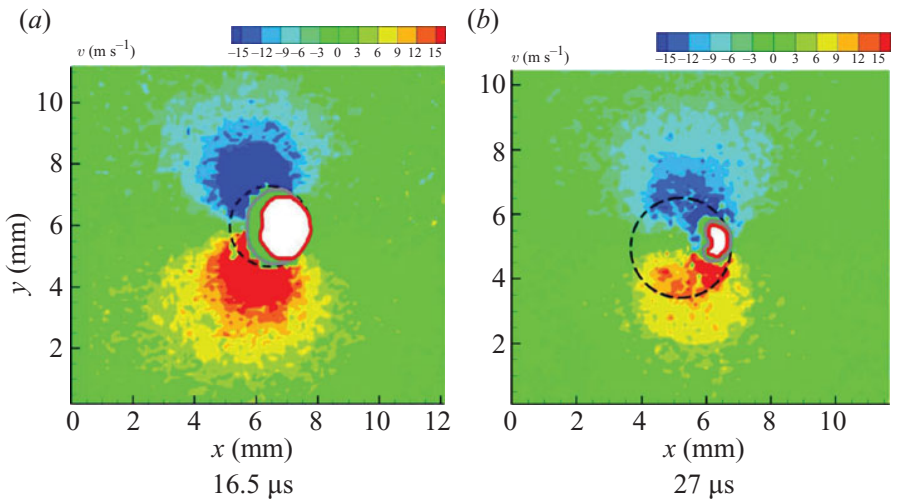

(c)

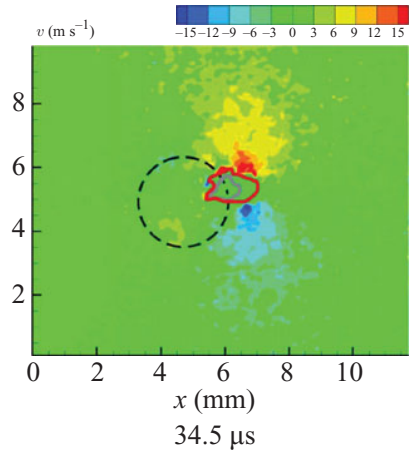

FIGURE 11. Examples of the velocity field induced by a collapsing void at selected times during the collapse. Times are referenced as in figure 8 . Initial void diameter is $3 \mathrm{~mm}$. Velocity contours in the range $\pm 15 \mathrm{~m} \mathrm{~s}^{-1}$ only are shown to emphasize the shape and extent of the region of influence.

\subsection{Single void region of influence}

Dear \& Field (1988) report that cavities having centres up to 2 diameters apart experience some degree of interaction. We use PIV data to provide a quantitative measure of the velocity induced by a single void collapse, examining the vertical velocity component. These plots reveal two circular regions above and below the void. Data obtained at different time delays after the beginning collapse are shown in figure 11. During the initial stages of collapse, the surrounding material is accelerated towards the cavity at velocities of up to $40 \mathrm{~m} \mathrm{~s}^{-1}$, approximately 1.5 times the projectile velocity. After the upstream interface has impacted the downstream interface, radial velocity vectors are observed downstream of the void.

The region of influence is defined as being the locus of points where the induced vertical component of the velocity is greater than $5 \%$ of the free-stream velocity. Measurements indicate that the void will influence material roughly 1.8-1.9 diameters away from the centre of the void. The consequence of this is that the velocity fields surrounding two voids will interact when their centres are at double this distance apart (3.6-3.8 void diameters). 


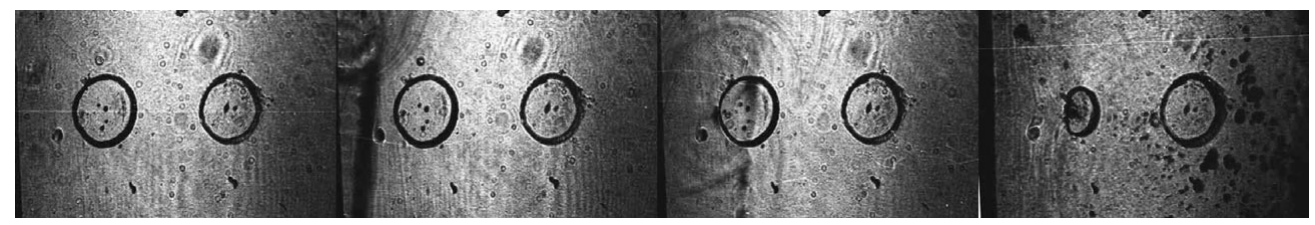

(a) $0 \mu \mathrm{s}$

(b) $3 \mu \mathrm{s}$

(c) $6 \mu \mathrm{s}$

(d) $19.5 \mu \mathrm{s}$

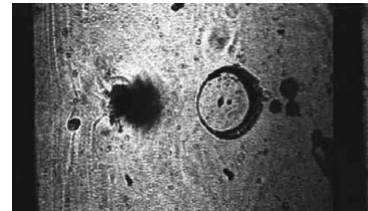

(e) $28.5 \mu \mathrm{s}$

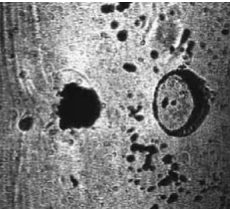

(f) $40.5 \mu \mathrm{s}$

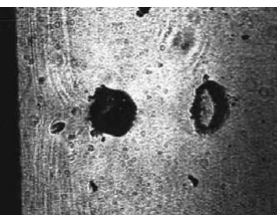

(g) $58.5 \mu \mathrm{s}$

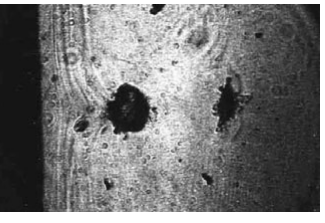

(h) $72 \mu \mathrm{s}$

FIGURE 12. High-speed images of a two void longitudinal array.

The evolution of the vertical velocity component as the collapse time increases is shown in figures $11(a)$ and 11(b). After jet impingement on the distal side of the void (figure 11c), the velocity fields above and below reverse direction as the fluid is now moving away from the void, in agreement with the radial velocity vectors observed in figure $10(c)$. The region of influence normalized by the void size measured from each image is $16.6,85.0$ and 42.6 for the three images in figure 11, respectively.

\section{Collapse of a two void longitudinal array}

Selected images from high-speed movies of the collapse of a longitudinal array of voids spaced 1 diameter apart are presented in figure 12. The time below each frame represents the time elapsed after figure 12(a). The arrival of the precursor wave occurs in figure $12(b)$. In figure $12(c)$, the wave has interacted with the upstream void. The semi-circular reflected wave can be seen in this frame on the upstream side of the upstream void. Additionally, there is evidence of a wave transmitted through the void in this frame. In figure $12(d)$, the high-speed jet in the upstream void is visible. The stress wave has now passed through the entire field of view, and the downstream void has not been affected; thus, the downstream void is 'shielded' by the upstream void. The collapse of the first void and subsequent pressure pulse is seen in figure $12(e)$. Figure $12(f)$ shows the beginning of the collapse of the downstream void, as well as some re-expansion of the upstream void. The remainder of the downstream voids collapse is captured in figures $12(\mathrm{~g})$ and $12(\mathrm{~h})$. A distinct jet is not observed to form in the downstream void; however, the collapse is still asymmetrical. The time to minimum diameter $t_{D, \min }$ of the upstream void is $21 \pm 1.5 \mu \mathrm{s}$, and the time to minimum volume $t_{V, \min }$ is $22.5 \pm 1.5 \mu \mathrm{s}$. These quantities are indistinguishable, $t_{D, \min }=t_{V, \min }=39 \pm 1.5 \mu \mathrm{s}$, for the downstream void.

Next, we examine the normalized plots of diameter versus time in figure $13(a)$ and volume versus time in figure $13(b)$ for the case of the longitudinal array. Diameter versus time data normalized by individual collapse times, as well as all data normalized by the collapse time of the upstream void, $t / t_{D, \min U S}$, are shown. The upstream void again exhibits an acceleration in the collapse profile for times up to $t / t_{D, \min } \approx 0.28$. This is followed by a period of linear collapse until the jet reaches the distal interface. The downstream void exhibits nearly similar behaviour when normalized by its own collapse time; however, acceleration is observed only until $t / t_{D, \min } \approx 0.12$. Note that 

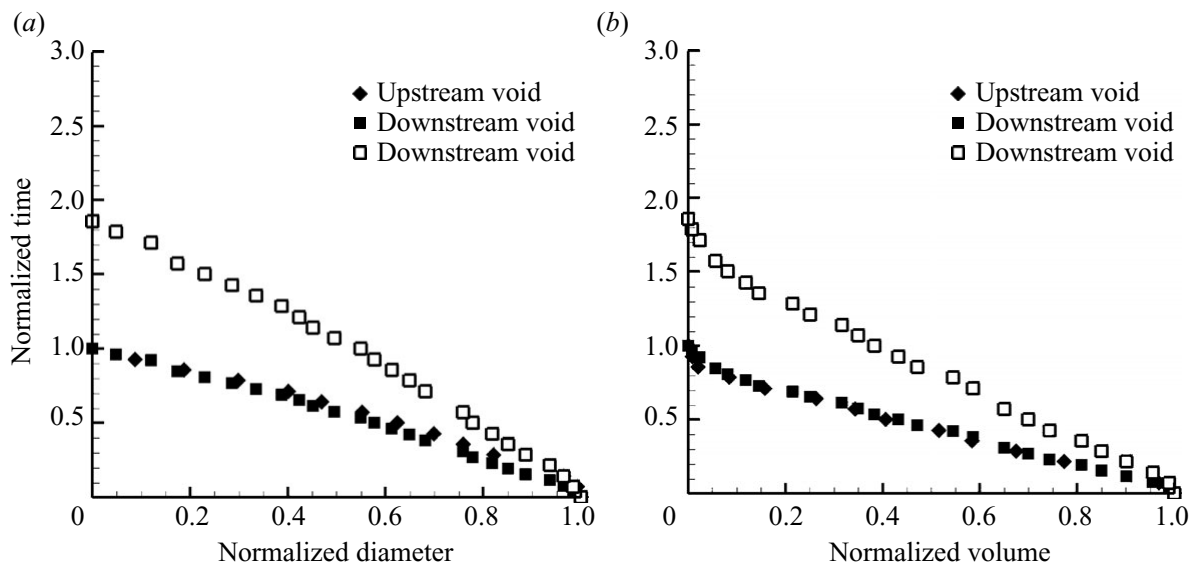

FIGURE 13. Normalized $(a)$ diameter and $(b)$ volume vs. time for a two void longitudinal array. Downstream void collapse data are normalized by the downstream void collapse time (solid symbols) and the upstream void collapse time (open symbols).

the collapse time of the downstream void is increased by a factor of 1.85 over the upstream void collapse time, indicating that the downstream void not only delays the collapse of the upstream void but also attenuates the strength of the loading stress wave.

The normalized volume versus time data exhibit very similar behaviour to the single void case, when each void collapse time is normalized by its respective $t_{V, \min }$. There is a period of acceleration at the beginning until $t / t_{V, \min } \approx 0.08$. The collapse then appears linear until $t / t_{V, \min } \approx 0.80$, when a deceleration is observed. Additionally, figure $13(b)$ presents the downstream void normalized by the time to minimum volume of the upstream void, $t / t_{V, \min }$. These data again demonstrate the increase in collapse time for the downstream void.

The planar velocity field data for the two void longitudinal array at four selected times are shown in figure 14. The first void interaction exhibits behaviour similar to the isolated void case, with loading wave diffraction similar to that observed in figure $10(a)$. Now, the second void is shielded from collapse in figure 14(b). It is apparent from the velocity contours that outside the shielded region, the stress wave has propagated beyond the second void location (figure 14b). When the upstream void internal jet impacts the distal interface, a pressure pulse is generated and propagates towards the downstream void. The collapse of the second void is triggered as seen in figure 14(c). By observing the velocity contours, we see that the local velocities in the inter-void region are higher than those in free-stream values. There will be a reflected expansion wave (which induces a velocity toward the void) when the upstream void pressure pulse interacts with the downstream void gel-gas interface. No internal jet is observed in the high-speed images; however, the void does collapse asymmetrically and radial velocity vectors are observed (figure 14d).

From the velocity contour plots in figure 14, data are extracted along selected cross-sections and plotted versus $x$ location in figure 15. Data are obtained at three cross-sections: one diameter above the void centreline, the void centreline and one diameter below the void centreline. Stress wave propagation into the downstream medium, as well as the evolution of the void interfaces, is observed in figure 15. The increase in velocity directly behind the void is seen in figure $15(a-c)$. Figure $15(d)$ 
(a)

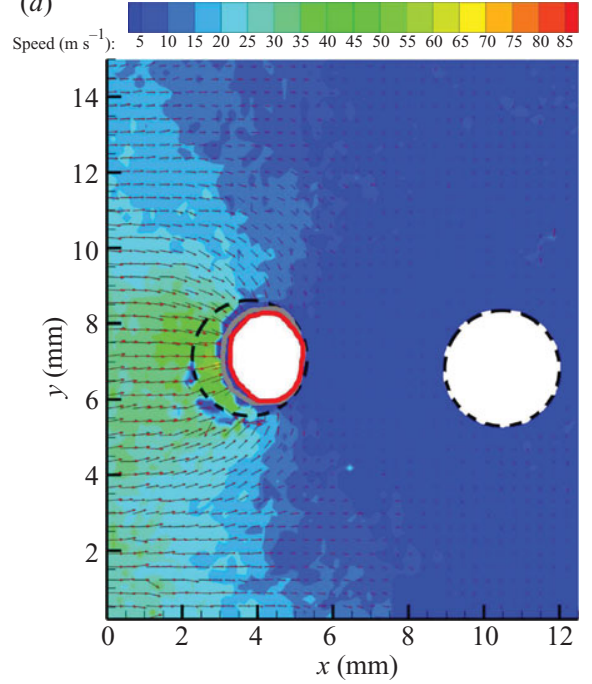

$16.5 \mu \mathrm{s}$
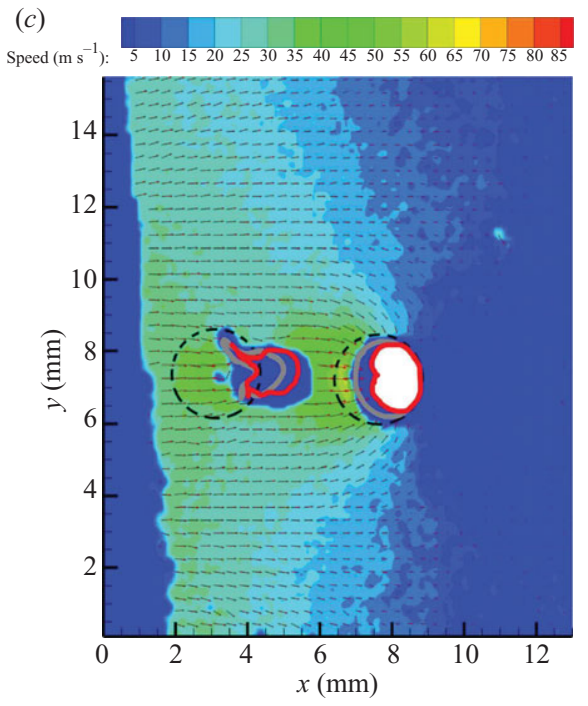

$48 \mu \mathrm{s}$
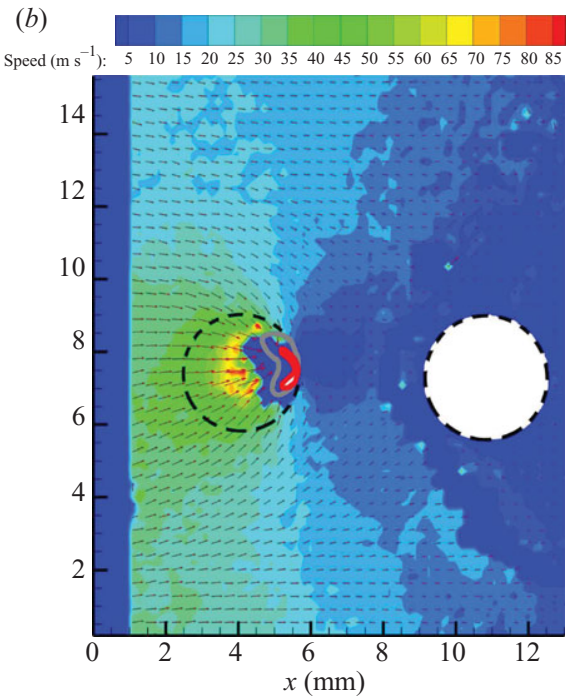

$25.5 \mu \mathrm{s}$
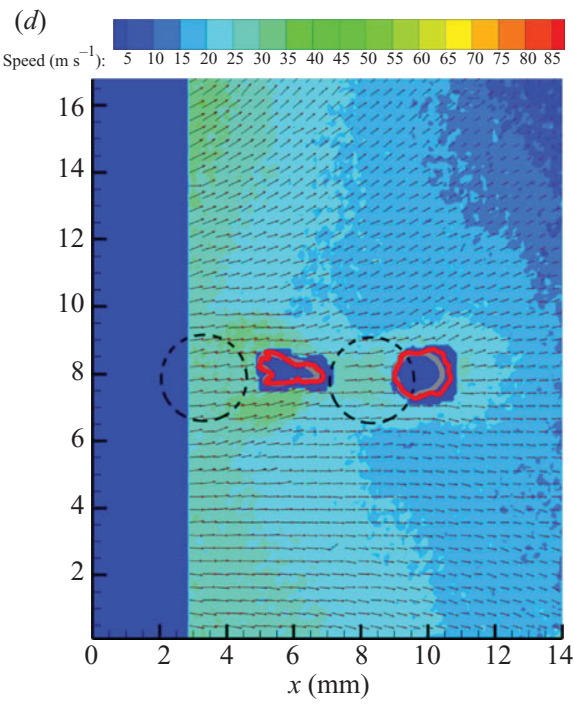

$66 \mu \mathrm{s}$

FIGURE 14. Velocity contours for a two void longitudinal array. Times are referenced from the same scale as in figure 12.

shows the post-collapse velocity profiles, where the velocity everywhere approaches the projectile velocity.

\section{Collapse of a four void staggered array}

The final configuration considered is a four staggered array. All voids are of $3 \mathrm{~mm}$ diameter. The voids are spaced such that the edges of any two adjacent voids are at one void diameter apart. The distance between the farthest upstream (west) voids and the farthest downstream (east) void is 1.83 diameters; thus, the centreline inter-void distance is greater than in the two void longitudinal configuration. Several frames of the collapse process for the staggered array are presented in figure 16. Times are 
(a)
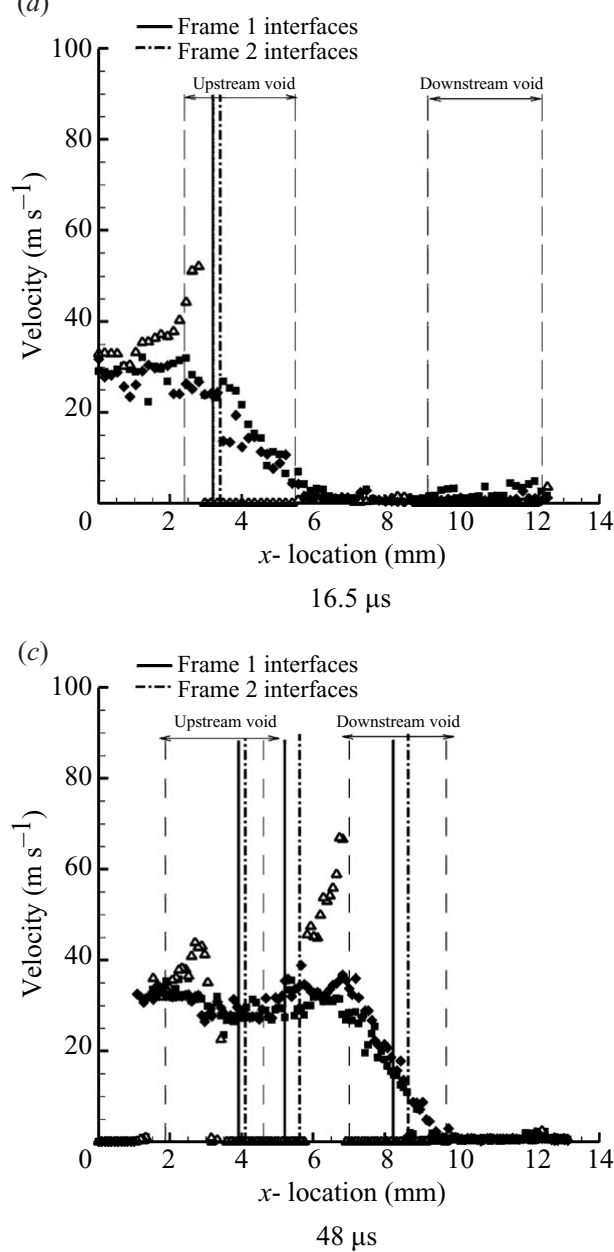
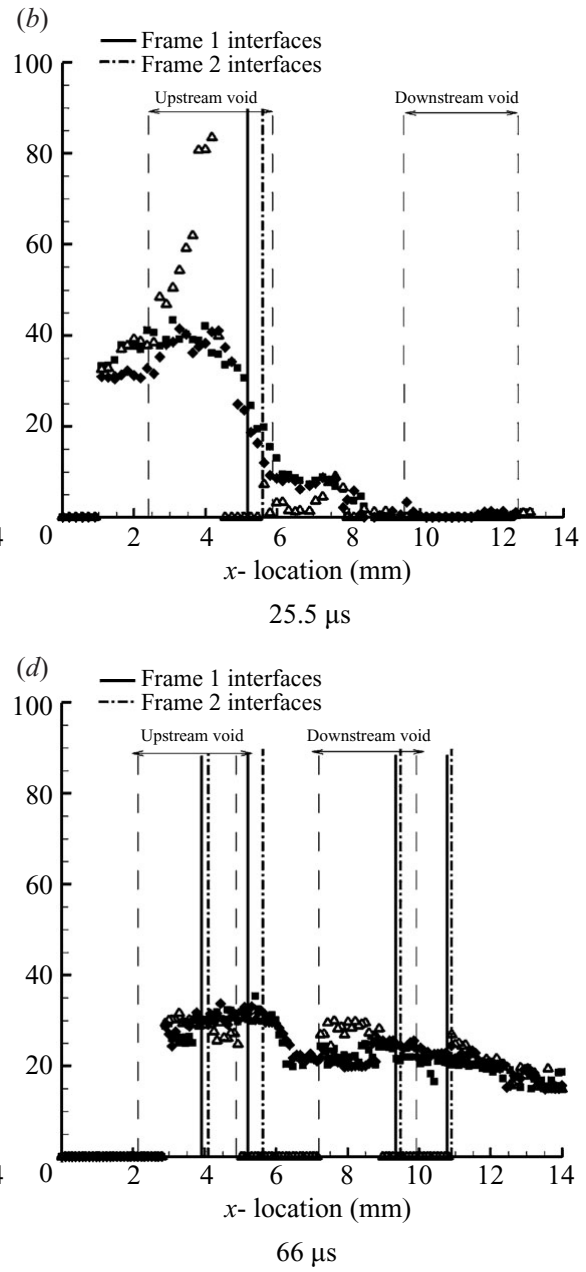

Figure 15. Velocity profiles for a two void longitudinal array. Times are referenced from the same scale as in figure 12. Data are obtained at three cross sections: above the centreline $(\bullet)$, on the centreline $(\triangle)$ and below the centreline $(\boldsymbol{\square})$.

referenced from figure $16(a)$. Figure $16(b)$ shows the precursor wave interacting with the west void. In figure $16(c)$, this wave has passed through the west void, generating a reflected wave; however, the west void has not begun to collapse. The beginnings of the jet in the west void can be seen in figure $16(d)$, becoming fully developed in figure 16(e). The collapse of the north and the south voids has begun in figure 16(e), illustrating that although the north and south voids are not directly shielded by the west void, the stress wave diffraction upon interaction with the west void results in a modified loading condition on the south and north voids. In the final stages of the collapse of the north and south voids, the interfaces undergo significant deformation (figure $16 g, h$ ). There is evidence of a jet formed in both the north and south voids. The east void has begun to collapse in figure $16(h)$, with the final stages captured in figure $16(i-l)$. No jetting is observed in the east void; however, the collapse is still asymmetrical. Collapse times $t_{D, \min }$ and $t_{V, \min }$ for the staggered array are presented in table 1 . The volume collapse times for all shielded voids are approximately increased 


$\begin{array}{lcccc}\text { Void } & \text { West } & \text { South } & \text { North } & \text { East } \\ t_{D, \min }(\mu \mathrm{s}) & 26 & 66 & 66 & 66 \\ t_{V, \min }(\mu \mathrm{s}) & 30 & 66 & 66 & 68\end{array}$

TABLE 1. Time to minimum diameter, $t_{D, \min }$, and time to minimum volume, $t_{V, \min }$, for each of the four voids. All times are $\pm 2 \mu \mathrm{s}$.

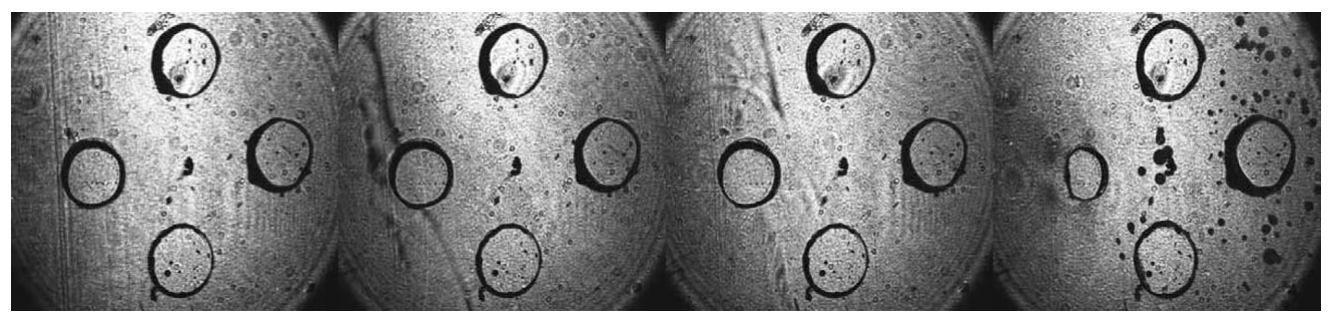

(a) $0 \mu \mathrm{s}$

(b) $4 \mu \mathrm{s}$

(c) $6 \mu \mathrm{s}$

(d) $20 \mu \mathrm{s}$

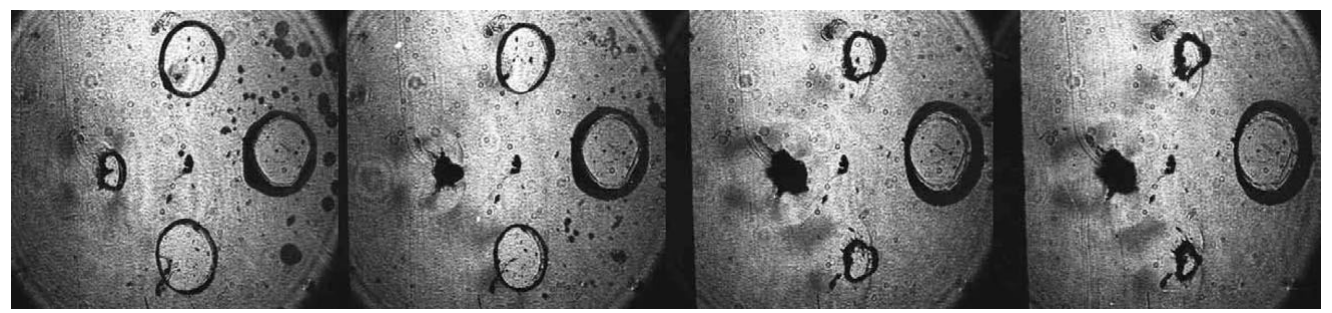

(e) $30 \mu \mathrm{s}$

(f) $46 \mu \mathrm{s}$

(g) $70 \mu \mathrm{s}$

(h) $76 \mu \mathrm{s}$

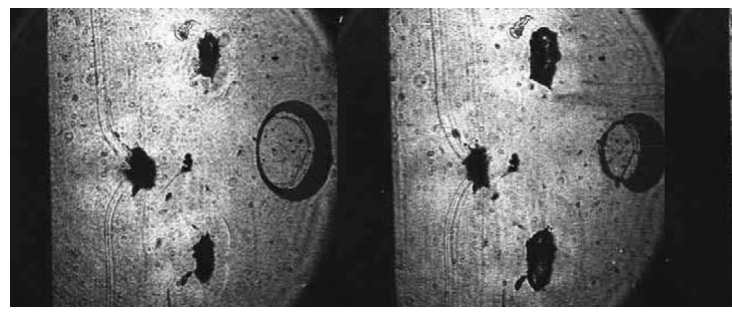

(i) $88 \mu \mathrm{s}$

(j) $112 \mu \mathrm{s}$

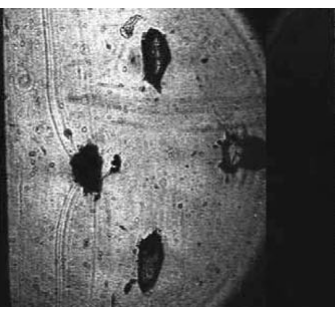

(k) $130 \mu \mathrm{s}$

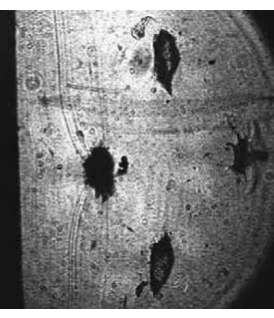

(l) $138 \mu \mathrm{s}$

FIGURE 16. High-speed images of the collapse of a four void staggered array. Initial void diameter is $3 \mathrm{~mm}$. Interframe time is $2 \mu \mathrm{s}$; selected frames are shown.

by a factor of 2 over the upstream (west) void collapse time, the diameter collapse times are increased by a factor of 2.5 .

Note that the east void exhibits some volumetric expansion before the collapse begins, illustrated by a dimensional diameter versus time plot in figure 17 . Time scale estimates show that this initial expansion may be attributed to the arrival of a reflected wave propagating from the end of the gel sample upon the incidence of the precursor pulse. Since the precursor velocity is substantially greater than the particle velocity, it was not possible to delay the reflected wave arrival until after the experimental time of interest in the four void case. The normalized distance and 


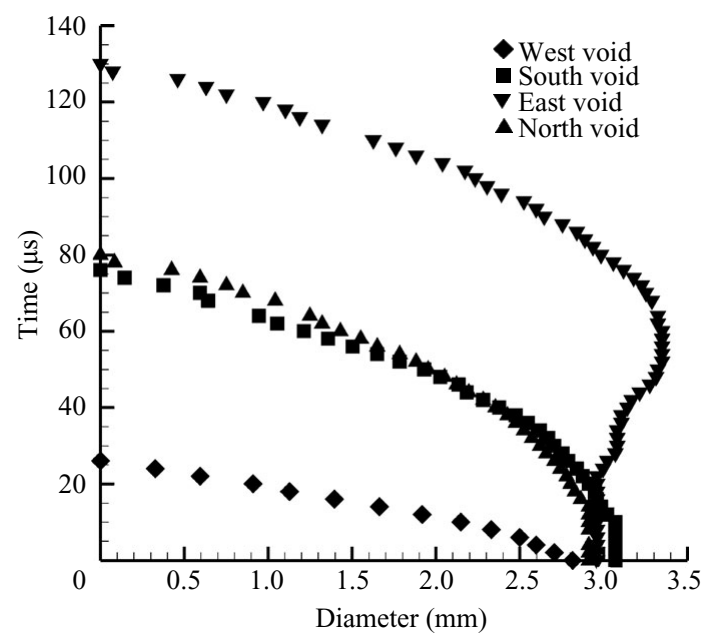

Figure 17. Dimensional plot of diameter vs. time for a four void staggered array.

volume histories for the east void show no subsequent effect because of the initial expansion.

The normalized plots of diameter versus time are shown in figures $18(a)$ and $18(b)$ and volume versus time are shown in figures 18(c) and 18(d). Data for each void are shown normalized by both the individual collapse time and the collapse time of the west void as a baseline case. In the plot of diameter versus time, when normalized by their respective collapse times, acceleration is observed at the beginning of the collapse process. However, for the three downstream voids, the region of acceleration appears to extend further in time than the upstream (west) void. The west void collapse becomes linear at $t / t_{D, \min } \approx 0.23$, while the downstream voids become linear at $t / t_{D, \min } \approx 0.45$. When all voids are normalized by the west void collapse time scale (figure $18 b$ ), collapse times are increased by a factor of about 2.5 with all three voids exhibiting a similar dynamic behaviour.

The normalized plots of volume versus time for the staggered array conform to the behaviour observed in the longitudinal array; there is a period of acceleration, followed by a period of linear collapse, and finally a period of deceleration. For the west void, the acceleration period extends to $t / t_{V, \text { min }} \approx 0.13$, followed by a period of linear collapse until $t / t_{V, \text { min }} \approx 0.86$. The north and south voids undergo acceleration until $t / t_{V, \min } \approx 0.41$ and linear collapse until $t / t_{V, \min } \approx 0.82$. The east void data show acceleration until $t / t_{V, \min } \approx 0.36$, followed by the linear collapse until $t / t_{V, \min } \approx 0.84$. Thus, the normalized duration of initial acceleration increases by a factor of 3 for both the vertically offset voids because of the loading wave diffraction about the west void. The threefold increase in the duration of the region of acceleration is also observed for the directly shielded (east) void. In all cases, the linear collapse period was maintained until the void reached $80-85 \%$ of the final volume, regardless of the duration of the initial acceleration or the location of the void.

PIV data at five selected times are shown in figure 19. Figure $19(a)$ is reminiscent of the single void case in which the material downstream of the west void is shielded; however, there is already wave interaction with the north and south voids with noticeable wave diffraction around the west void as well as around the north and south voids. The final stages of the west void collapse, together with substantial north and south void collapse, are evident in figure 19(b). The inter-void shielded region 
(a)

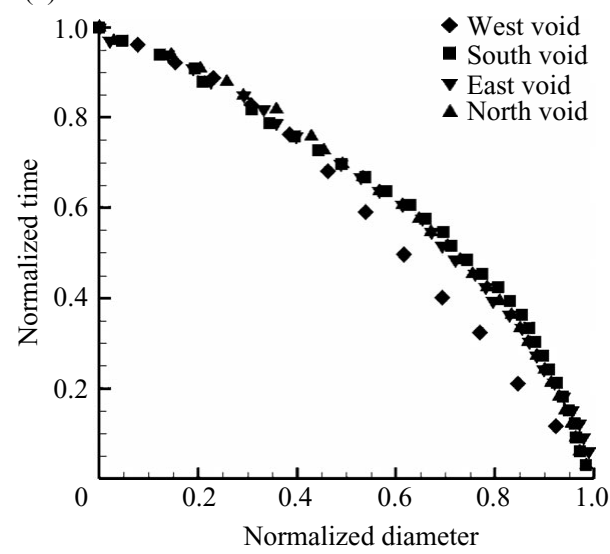

(b)

(d)

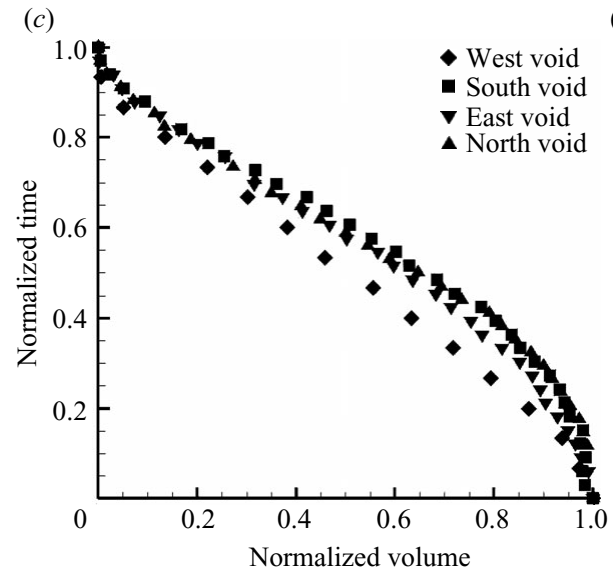

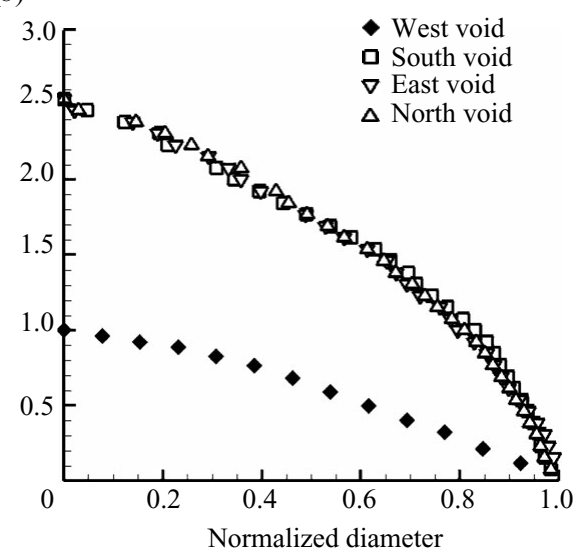

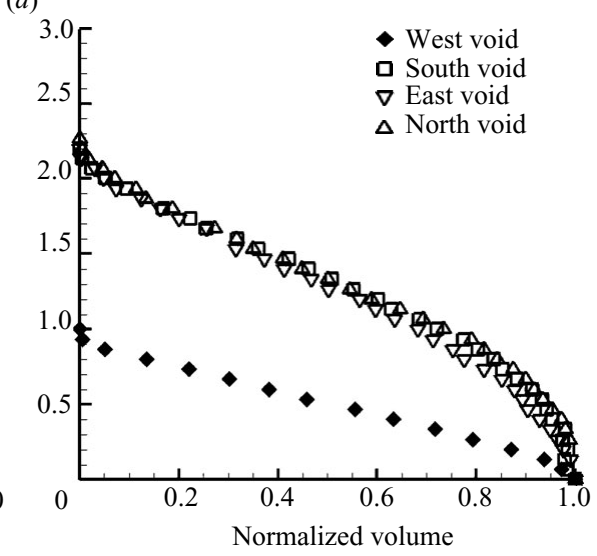

FIGURE 18. Normalized diameter $(a, b)$ and volume $(c, d)$ volume vs. time for a four void staggered array. $(a, c)$ The data normalized by the individual collapse time; $(b, d)$ the data normalized by the west void collapse time.

has become smaller. The west void collapse and the subsequent wave velocity field in the interior of the array are shown in figure 19(c). The vectors indicate an interaction of the west void with the north and south voids with velocity vectors diverging from the centreline towards the two outer voids. This same effect is seen in figure $19(d)$. In this case, the north and south void collapse results in flow towards the east void. The post-collapse velocity field around the east void is shown in figure 19(e). The void has collapsed asymmetrically, and a radially expanding velocity field downstream of the collapsed void is again observed.

As in the case of the four void array, several velocity profiles are extracted along selected cross-sections plotted versus $x$ location in figure 20. Indicators of void interface location have not been included in these plots for clarity, but can be referenced from figure 19. As the collapse process is very nearly symmetric about the array centreline, four cross-sections are selected: the east void centreline, the south void centreline, the midline between the previous two, and one diameter below the south void centreline.

Figures $20(a)$ and $20(b)$ show the west void collapse and the start of the south void collapse. The velocity profile (along the west void centreline) resulting from the 
(a)

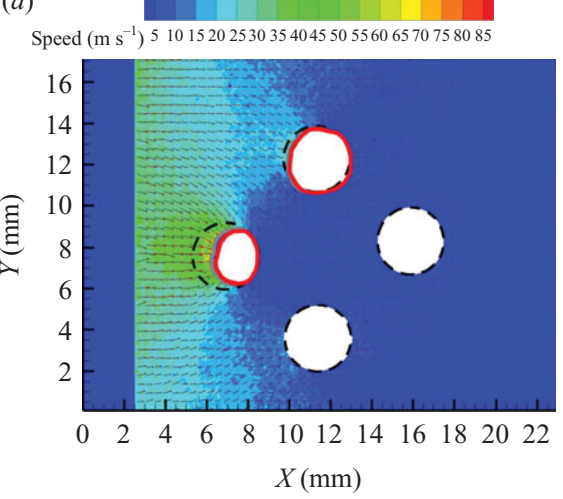

$16 \mu \mathrm{s}$

(c)

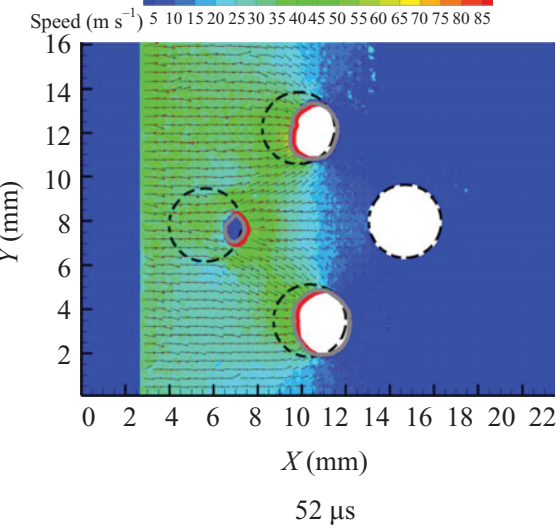

(b)

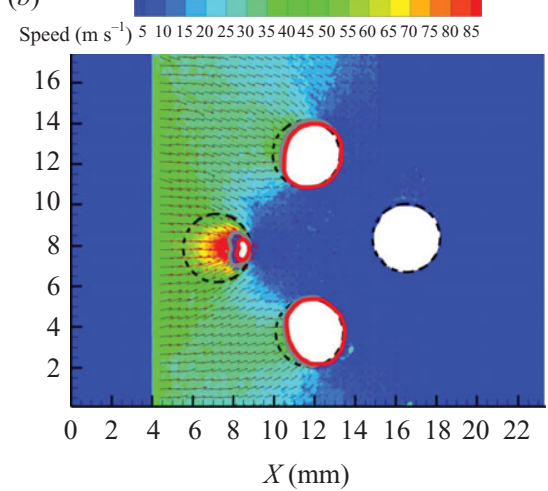

$32 \mu \mathrm{s}$

(d)

Speed $\left(\mathrm{m} \mathrm{s}^{-1}\right)^{5} 10152025303540455055606570758085$

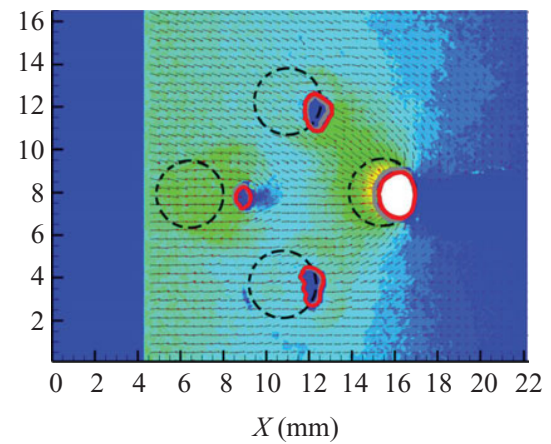

$108 \mu \mathrm{s}$

(e)

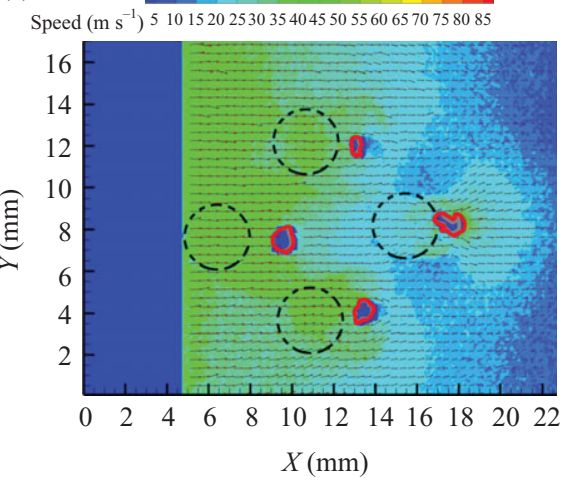

$140 \mu \mathrm{s}$

FIGURE 19. Velocity profiles for a four void staggered array. Times are referenced from the same scale as in figure 16.

impact of the west void internal jet has a similar shape to the velocity profile along the midline (figure 20c). There is a large velocity increase from nearly zero to $75 \mathrm{~m} \mathrm{~s}^{-1}$ on the upstream interface of the east void (figure 20d). Lastly, figure 20(e) shows the post-collapse velocity field; again, a radially expanding velocity field downstream of the east void is observed. 

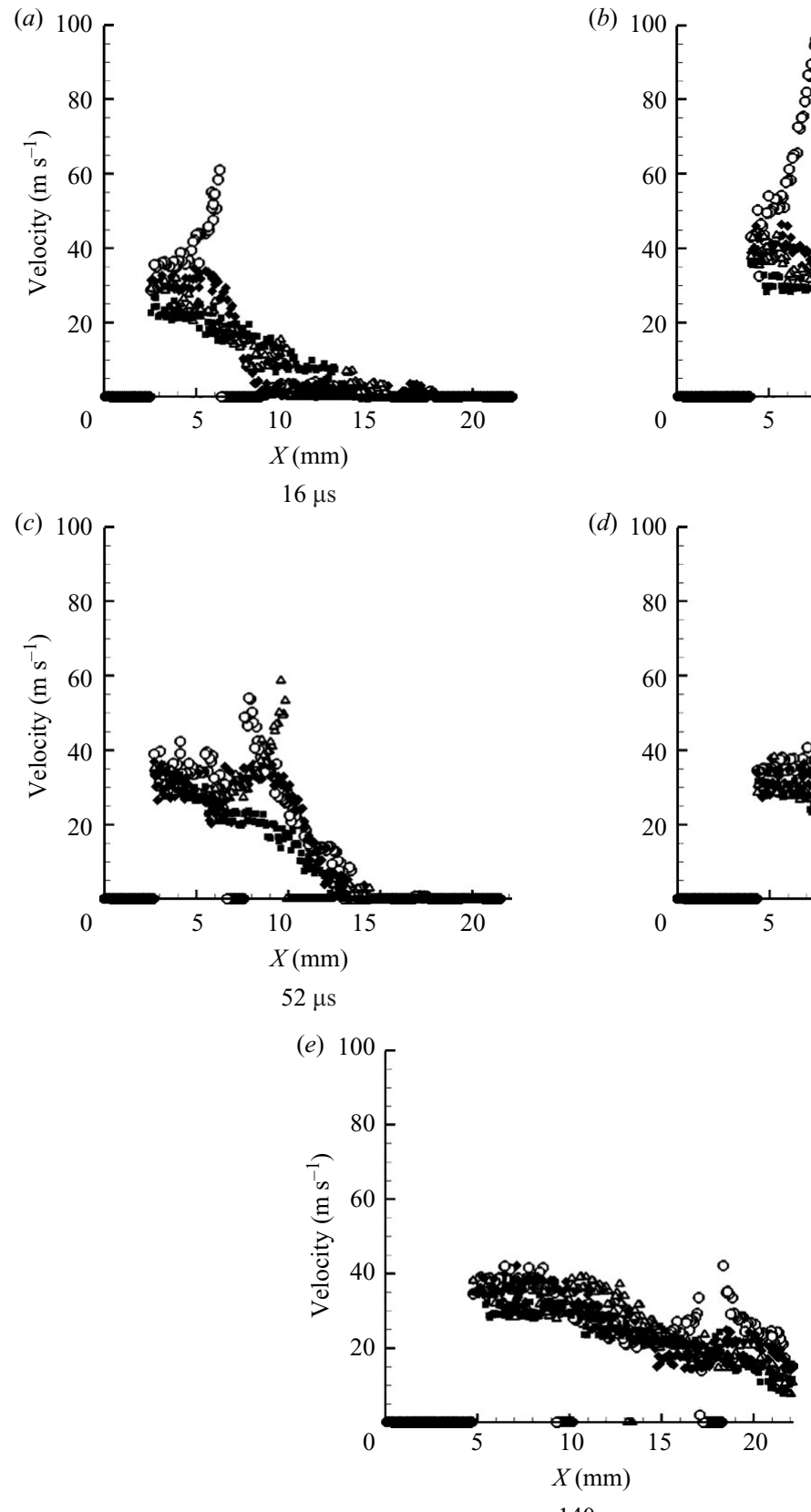

$140 \mu \mathrm{s}$ (b)
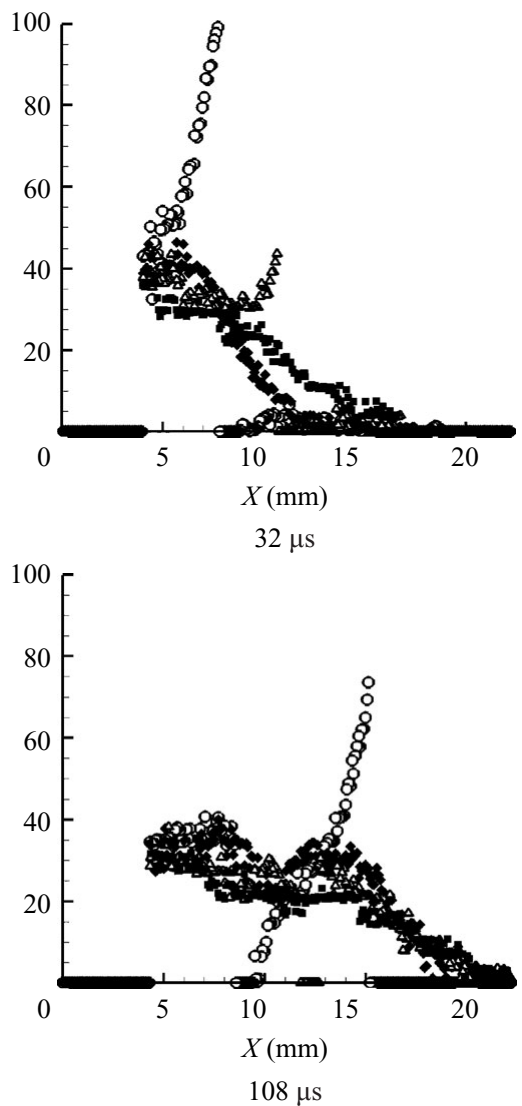

FIGURE 20. Velocity cross-sections for four void staggered array. Times referenced are the same as in figure 12. Data are at four selected cross-sections: west void centreline $(\bigcirc)$, array midline $(\bullet)$, south void centreline $(\triangle)$ and 1 diameter below the south void centreline $(\mathbf{\square})$.

\subsection{Interaction in a four void staggered array}

To quantify the interactions of the four staggered array, we again utilize the vertical component of the velocity and observe deviations from the case of the single void profiles. These data are shown for varying times in figure 21 . 


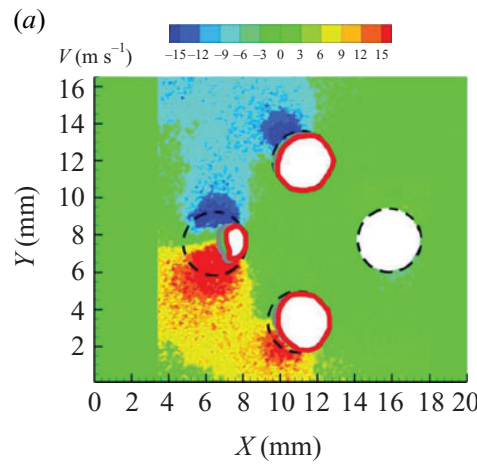

$24 \mu \mathrm{s}$

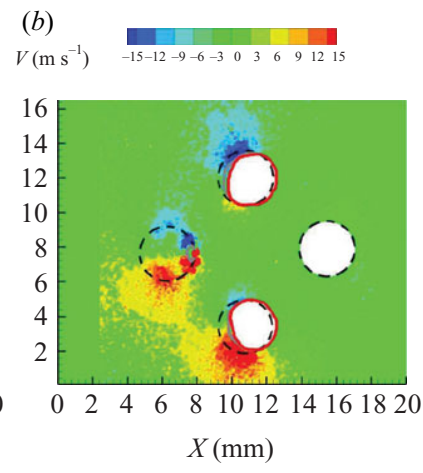

$30 \mu \mathrm{s}$

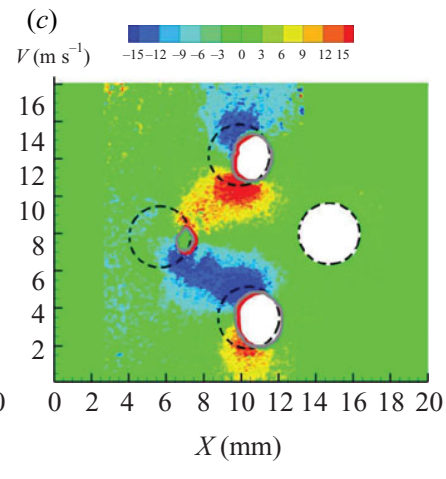

$54 \mu \mathrm{s}$

FIGURE 21. Examples of the velocity field induced by a collapsing four void staggered array, at selected times during the collapse. Times are referenced as in figure 16. Initial void diameter is $3 \mathrm{~mm}$. Velocity contours in the range $\pm 15 \mathrm{~m} \mathrm{~s}^{-1}$ only are shown to emphasize the shape and extent of the region of influence.

Figure 21(a) shows the velocity field around the west void, which is qualitatively similar in size and shape to single void profiles presented in figure 11( $a)$. The north and south voids also have an induced field; however, it is asymmetrical, which is expected from the high-speed images and PIV data above. These two velocity fields exhibit distinct interaction as predicted in $\S 4.1$, as the voids are spaced 2 diameters centre to centre. This plot indicates that each void simultaneously influences the velocity field of the other void; therefore, downstream voids in this loading condition may, in fact, influence the upstream voids. Figure 21(b) shows a decrease of the region around the west void, which agrees with figure 11. The north and south voids have now developed profiles on inside lower and upper sides, respectively; however, the profiles are still highly asymmetrical. Figure 21(c) shows the velocity fields around the north and south voids after the collapse of the west void. Again, there is a distinct interaction between resultant velocity field from west void collapse with the north and south voids, which appears to be a stronger interaction than the one seen in figure $21(a)$. The velocity fields surrounding the north and south voids exhibit different profiles than the single void. The upper and lower lobes of velocity surrounding each void display asymmetries in shape and location in comparison to the single void profiles.

\section{Comparison of stress wave and shock-wave loading}

In this study, we examine the interaction of a stress wave, such as might result from an accidental mechanical insult, with void arrays. In contrast to a shock wave, the stress wave profile has a length scale that is comparable to both the void diameter and the inter-void spacing. We compare the internal volume history obtained in the present experiments under stress wave loading with literature data for shock-induced cavity collapse (figure 22). Bourne \& Field (1992) experimentally investigated shockinduced collapse of cylindrical, air-filled voids in gelatine, with cavity diameters from 3 to $12 \mathrm{~mm}$ and a shock pressure ratio of $0.26 \mathrm{GPa}$. The experimental data from Haas \& Sturtevant (1987), who examined the shock collapse of cylindrical helium and Refrigerant 22 gas bubbles in air, are also shown. Bourne and Field found a linear curve fit to their data over the entire collapse regime. These data, however, show that linear behaviour occurs only over a portion of the collapse, with regions of acceleration and deceleration at the beginning and end of the collapse history. 


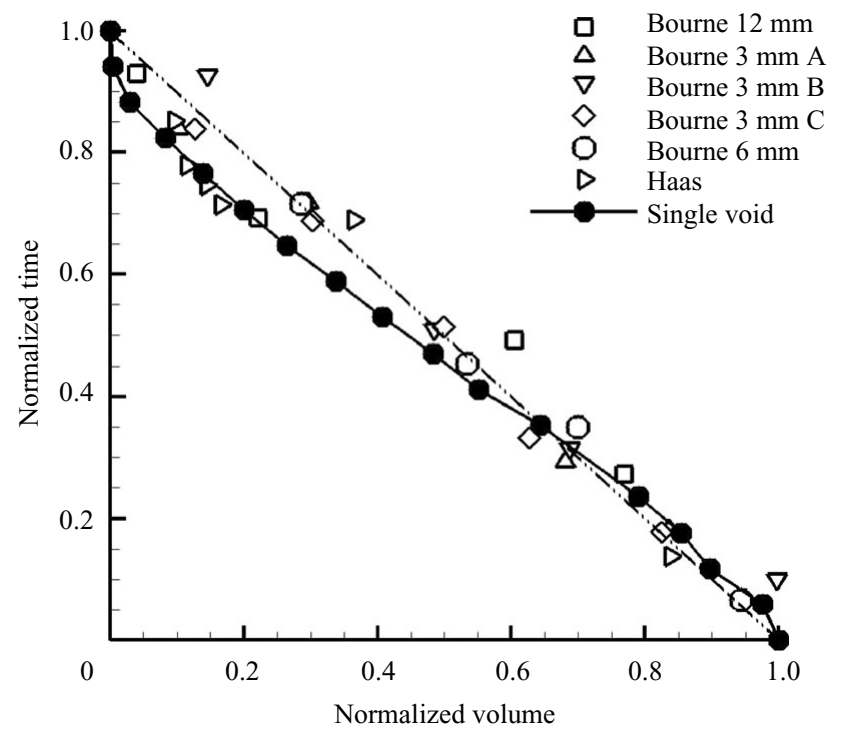

FIGURE 22. Plots of normalized volume vs. normalized time for cylindrical voids. Collapse times are normalized by $t_{V, \min }$. Current data (single void) are compared with experiments of Bourne \& Field (1992) and Haas \& Sturtevant (1987). (A connecting line between current data points is shown for clarity.) The linear curve fit of Bourne \& Field (1992) is also included.

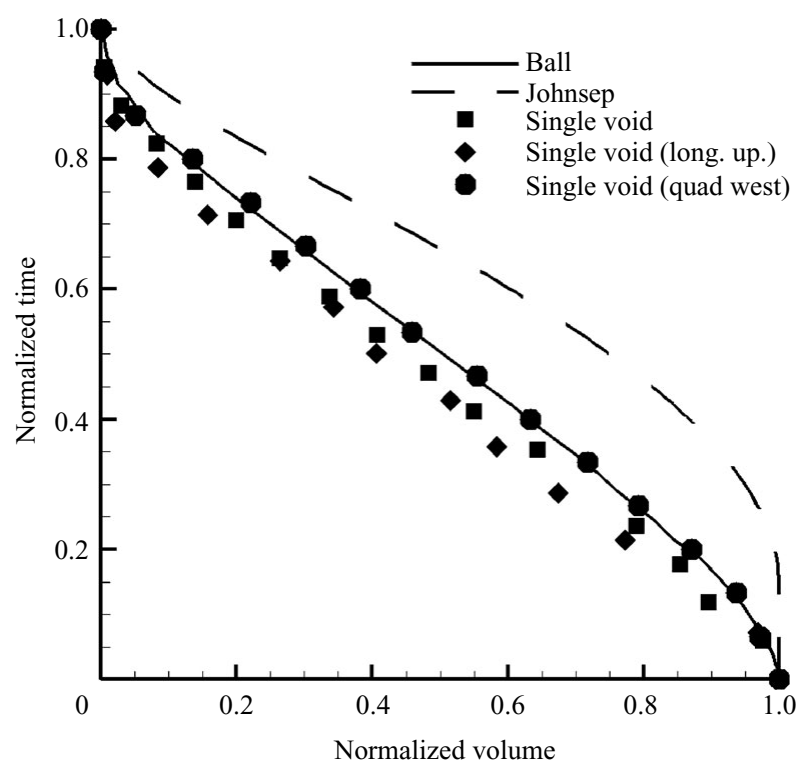

FIGURE 23. Plots of normalized volume vs. normalized time for current experimental data and literature simulations from Ball et al. (2000) (two-dimensional) and Johnsen \& Colonius (2009) (three-dimensional). Collapse times are normalized by $t_{V, \min }$. Good agreement is obtained between experiments and two-dimensional numerical collapse histories.

The present experimental data are also compared with numerical collapse histories (figure 23). Ball et al. (2000) carried out simulations of the Bourne \& Field (1992) shock-induced collapse experiments and reported the initial and final acceleration and deceleration regions. These numerical data show very good agreement with the present 
experiments over the entire collapse history, with the initial and final stages captured in both the experiments and simulations. Ball et al. (2000) report a linear collapse region between $0.27<t / t_{V, \min }<0.81$, which agrees well with the current results $(\S 4)$. The two-dimensional data are in good agreement despite the difference in the loading wave, indicating that the pressure ratio is still the dominant parameter in determining the collapse dynamics at these conditions.

Also shown for reference are data from simulations by Johnsen \& Colonius (2009) of the shock-induced collapse of a spherical air bubble in water. Initial acceleration and final deceleration are also observed in these simulations. As expected, the initial volume reduction is slower and the final volume reduction is faster in the spherical case than in the cylindrical case.

In summary, the present internal volume history is in good agreement with the two-dimensional simulations of Ball et al. (2000), despite stress versus shock-wave loading. At the current conditions, the loading wave profile has no evident effect on the collapse history of an isolated cavity. Collapsing cylindrical cavities undergo initial acceleration and final deceleration, a qualitatively similar behaviour to collapsing spherical voids.

Next, we examine cavity collapse times across a range of pressure ratios. The Rayleigh collapse time $t_{c}$ of symmetrical, spherical bubble scales with the square root of the pressure difference (Rayleigh 1917; Brennan 2005)

$$
t_{c} \sim 0.915\left(\frac{\rho}{p_{\infty}-p_{v}}\right)^{0.5} R_{i},
$$

where $\rho$ is the density in the surrounding medium, $p_{\infty}$ is the pressure at infinity, $p_{v}$ is the internal pressure and $R_{i}$ is the initial bubble radius. A correction for asymmetric collapse (near a wall) introduced the distance to the wall as a parameter but retained the same pressure and initial bubble radius dependence (Rattray 1951). Johnsen \& Colonius (2009) compare the time to minimum volume for Rayleigh and shock-induced collapse of spherical bubbles over a range of pressure ratios up to 714 . Shock-induced collapse times are approximately one time unit greater than Rayleigh collapse, an effect attributed to the shock propagation time across the bubble. Of relevance to the current study, Johnsen \& Colonius (2009) find the same collapse time scaling with pressure ratio for both shock-induced and Rayleigh collapse, despite asymmetric collapse and internal jet formation under shock loading.

For cylindrical bubbles, no analogue to the Rayleigh equation exists. Small and large amplitude oscillations can be modelled using a wave equation (Epstein \& Keller 1972), but to the authors' knowledge, no collapse time prediction model is available. We compare the time to minimum volume $t_{V, \min }$ from the present study with a compilation of cylindrical bubble collapse times from the literature as a function of the pressure ratio $p / p_{o}$ across the loading wave (figure 24). Note that $t_{V, \min }$ is normalized by the initial bubble radius $R_{i}$ and the fluid sound speed $c$.

To achieve a range of loading wave pressure ratios, high-speed movie collapse time measurements were made with projectile velocities of 16,27 and $78 \mathrm{~m} \mathrm{~s}^{-1}$, corresponding to calculated pressure ratios of 249, 421 and 1140. A power-law curve fit to the current data is shown. Perhaps surprisingly, the exponent is close to the -0.5 dependence of the Rayleigh curve. Data from the two-dimensional simulations of Ding \& Gracewski (1996), Ball et al. (2000), Hu \& Khoo (2004) and Sushchikh \& Nourgaliev (2005) for shock loading conditions are shown. Ding \& Gracewski (1996) identify a time of maximum pressure for symmetric collapse and a time of jet 


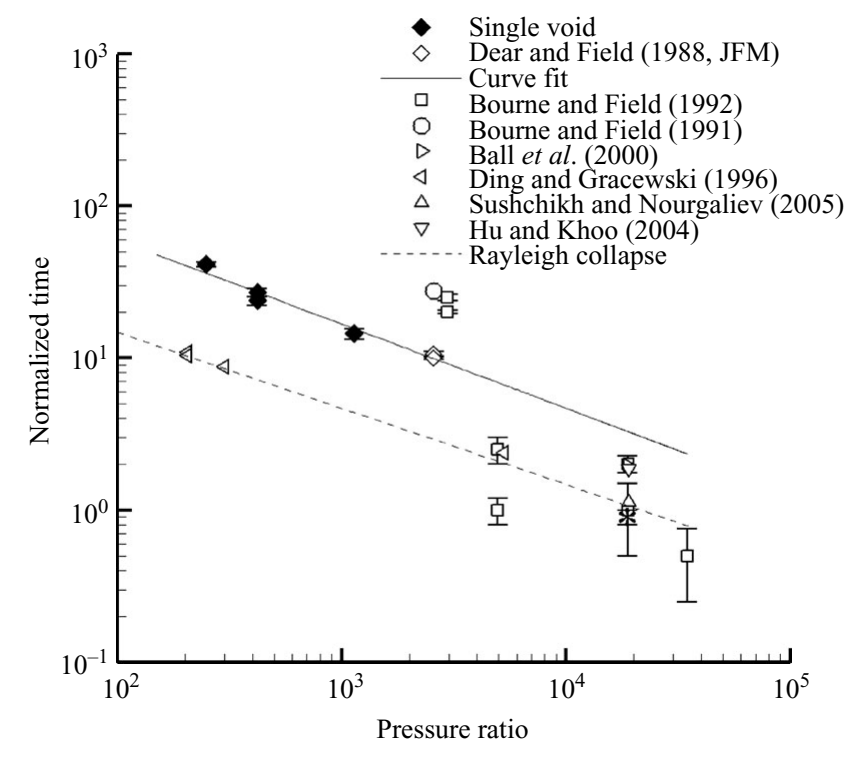

FIGURE 24. Normalized collapse time vs. loading wave pressure ratio for single, cylindrical voids. A power-law curve fit, $t_{V, \min } c / R_{i}=710\left(p / p_{o}\right)^{-0.55}$, is shown to the current data $(\diamond)$ and Dear \& Field (1988) $(\diamond)$.

impingement for asymmetric collapse. Ball et al. (2000), Hu \& Khoo (2004) and Sushchikh \& Nourgaliev (2005) report a time to minimum volume. Although the internal volume histories are very similar to current results (figure 23), the collapse times are significantly different. Experiments show increased collapse times over the numerical results. Data from the two-dimensional simulations of Ding \& Gracewski (1996) also have pressure dependence close to the Rayleigh curve for three-dimensional gas bubbles in water.

The experimental data of Dear \& Field (1988), Bourne \& Field (1991) and Bourne \& Field (1992) for cylindrical voids under shock loading are also presented. In spite of the similarity in the experimental set-up, there is not good agreement between these data and the present study in all cases. The data from Dear \& Field (1988) appear consistent with our data and are included in the curve fit. However, collapse times from the related studies of Bourne and Field are significantly different. Simulations by Ball et al. (2000), Hu \& Khoo (2004) and Sushchikh \& Nourgaliev (2005) were carried out at two selected conditions from Bourne \& Field (1992) experiments as a code validation exercise. The simulations of Ball et al. (2000) and Hu \& Khoo (2004) agree, but differ by a factor of 2 from the experimental value (indicated by an asterisk in figure 24). In fact, the $6 \mathrm{~mm}$ void diameter numerical collapse times correspond to the $12 \mathrm{~mm}$ void diameter experiment. The $12 \mathrm{~mm}$ void diameter simulations of Sushchikh \& Nourgaliev (2005) correspond to the $6 \mathrm{~mm}$ void diameter experiment. A shorter collapse time is obtained numerically for the $12 \mathrm{~mm}$ diameter void than that for the $6 \mathrm{~mm}$ diameter void at the same pressure ratio.

From spherical bubble collapse simulations, Johnsen \& Colonius (2009) report shock-induced collapse time dependence on pressure ratio is close to the Rayleigh collapse. A similar conclusion is reached in this work for cylindrical cavities. Under stress wave loading, experimental collapse times scale with pressure ratio with an exponent that is in good agreement with Rayleigh collapse. Under shock-wave loading, 


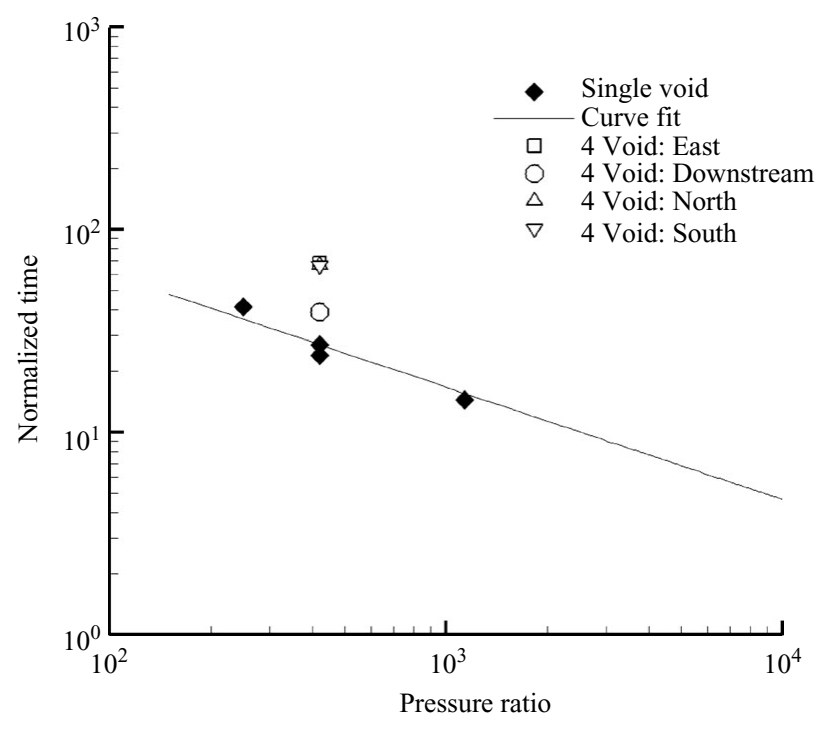

FIGURE 25. Normalized collapse time, $t_{V, \min } c / R_{i}$, vs. pressure ratio for different void locations.

simulations by Ding \& Gracewski (1996) also follow Rayleigh scaling. This is perhaps reasonable given that a comparison of present collapse history data with numerical simulations shows that at the conditions of this study, the dynamic behaviour of collapse under stress wave loading and shock-wave loading is similar. Thus, Rayleigh collapse appears to provide a reasonable model for the scaling of collapse time with pressure ratio for both cylindrical and spherical cavities under shock and stress wave loading.

Wave diffraction around the void arrays significantly affects the loading condition and the collapse times of the individual voids. Collapse times for voids in longitudinal and staggered arrays are increased over the single void case. To illustrate this effect, collapse times for shielded and offset voids are compared with isolated void collapse times (figure 25). If a void is located directly downstream of another void, an $85 \%$ increase in collapse time is observed. Voids that are vertically offset are also affected, with collapse time increasing by a factor of 2 . Results suggest that a modified pressure ratio should be used in predicting the collapse times for interacting arrays.

\section{Conclusions}

We investigate the dynamic interactions of multiple collapsing cavities under stress wave loading, such as occurs for example in energetic materials in accident scenarios with low impact velocity mechanical insult. Hotspot formation and detonation initiation involves complex thermochemical, fluid and structural processes over an extremely broad range of scales. We experimentally study a model problem with arrays of cylindrical voids in a gelatinous material. These results, therefore, also have relevance to void collapse in hydraulic and biomedical applications. A single void and two configurations of void arrays are examined: a two void longitudinal array and a four void staggered array. High-speed movies provide time-resolved visualization of the collapse process, in tandem with PIV measurements in the surrounding media at selected stages during the collapse. 
In the baseline case of a single void, we observe asymmetric collapse and the formation of a central internal jet. The high-speed jet impinges upon the stationary distal void interface and generates a pressure pulse followed by a radially expanding velocity field. Neighbouring voids have been observed to interact when located approximately 1 diameter apart in previous studies. We quantify a void collapse region of influence by measuring the extent of the vertical velocity field as a function of time.

In a two void longitudinal array, both collapse-shielding and collapse-triggering occur. The upstream void is observed to collapse in the same manner as the single void. However, the stress wave diffracts around the upstream void, and the downstream void collapse is delayed by this shielding effect. The downstream void collapse is subsequently triggered by the pressure pulse generated by the upstream void central jet impingement. The downstream void exhibits no distinct jetting, but does collapse asymmetrically.

In the case of the four void staggered array, the upstream void collapses again in a similar manner to the single void case. The second column of voids tends to angle slightly inward towards the fourth void. The collapse of the upstream void triggers a pressure pulse followed by a velocity field that are very similar to those produced by the other configurations, implying a similarity in the characteristics of the collapse regardless of the level of shielding. The collapse of the last void appears to be more strongly dependent on the middle column of voids, rather than on the upstream void. Normalized diameter versus time plots show similar collapse dynamics for the offset voids and the downstream void.

Internal volume and centreline diameter histories during the collapse are reported. In contrast to the entirely linear behaviour reported in previous experiments, the present internal volume versus time data show an initial acceleration of the upstream interface, followed by linear collapse and a final deceleration. Very good agreement is obtained with two-dimensional numerical simulations, which also capture the regions of acceleration and deceleration in the collapse history. These profiles are qualitatively similar to curves obtained from three-dimensional simulations. Centreline diameter data show an initial acceleration followed by a linear regime, but no final deceleration due to the formation of the central jet. The experimental volume history data for the single void under stress wave loading are in good agreement with existing numerical simulations of a void under shock-wave loading, indicating that at the conditions of the study, the pressure ratio is the dominant parameter in determining the collapse dynamics.

For a spherical bubble, the Rayleigh collapse time scales with the square root of the pressure difference. The collapse time for spherical bubbles under shock loading has been previously shown to have pressure ratio dependence similar to Rayleigh collapse. No direct analogue for the Rayleigh-Plesset equation exists for cylindrical bubbles. We compare time to minimum volume results from the present study with existing numerical and experimental data. Normalized collapse time is generally found to increase with decreasing pressure ratio. A power law can be fit to the experimental data from the present study and Dear \& Field (1988), and an exponent of -0.55 is obtained. Experimental data from the related studies of Bourne \& Field (1992) do not fall on this curve. Simulations universally underpredict experimental collapse times but appear to show a similar pressure dependence as experiments. These results indicate that, as for spherical bubbles, the collapse times of dynamically loaded cylindrical cavities have pressure ratio dependence close to Rayleigh collapse. In summary, Rayleigh collapse appears to be a reasonable model for the scaling of 
collapse time with pressure ratio for both cylindrical and spherical cavities under shock and stress wave loading for the conditions of this study.

In addition to inter-void collapse-shielding and collapse-triggering interactions, the presence of multiple voids affects the local loading condition. Wave diffraction is observed and found to have a significant effect on the collapse time. This has implications for detonation initiation, when ignition spread occurs under critical conditions of voids collapsing in concert. In a longitudinal array, the downstream void collapse time is increased $85 \%$ over the upstream void collapse time. In a staggered array, the diameter collapse time increases by a factor of 2.5 and the volume collapse time increases by a factor of 2 . Results suggest that a modified loading wave pressure ratio should be used in predicting the collapse times for interacting void arrays.

The authors gratefully acknowledge Professor John Lambros for the generous loan of laboratory equipment and space which made this study possible. We thank Matthew Parker for his initial experiments, Professor Greg Elliott for valuable input in the PIV measurements, Professor Jonathan Freund, Dr Ratnesh Shukla, Professor Carlos Pantano and Professor Scott Stewart for useful discussions comparing experiments and simulations, and researchers in the DE-9 division at Los Alamos National Laboratories. We also thank Dr Eric Johnsen and Professor Tim Colonius for sharing their numerical data. This work was supported in part by the US Department of Energy through the University of California under subcontract B523819.

\section{REFERENCES}

BAER, M. R. 2002 Modelling heterogeneous energetic materials at the mesocale. Thermochimica Acta 384, 351-367.

Bailey, M. R., Crum, L. A., Evan, A. P., McAteer, J. A., Williams, J. C., Sapozhnikov, O. A., Cleveland, R. O. \& Colonius, T. 2003 Cavitation in shock wave lithotripsy. In Fifth Intl Symp. on Cavitation. Osaka.

Ball, G. J., Howell, B. P., Leighton, T. G. \& Schofield, M. J. 2000 Shock-induced collapse of a cylindrical air cavity in water: a free-Lagrange simulation. Shock Waves 10, 265-276.

Bourne, N. K. \& Field, J. E. 1990 Collapsing cavities in reactive and non-reactive media. In 19th Intl Congress on High-Speed Photography and Photonics SPIE, vol. 1358, pp. 1046-1055. International Society for Optical Engineering.

Bourne, N. K. \& Field, J. E. 1991 Bubble collapse and the initiation of explosion. Proc. Math. Phys. Sci. 435 (1894), 423-435.

Bourne, N. K. \& Field, J. E. 1992 Shock-induced collapse of single cavities in liquids. J. Fluid Mech. 244, 225-240.

Bourne, N. K. \& Field, J. E. 1999 Explosive ignition by the collapse of cavities. Proc. R. Soc. A 455, 2411-2426.

Bourne, N. K. \& Milne, A. M. 2003 The temperature of a shock-collapsed cavity. Proc. R. Soc. A 459, 1851-1861.

Bowden, F. P. \& Yoffe, A. D. 1952 Ignition and Growth of Explosion in Liquids and Solids. Cambridge University Press.

Brennan, C. E. 2005 Fundamentals of Multi-Phase Flow. Cambridge University Press.

Campbell, A. W. \& Travis, J. R. 1985 Shock desensitization of PBX-9404 and composition B-3. In Proc. 8th Symp. (Intl) on Detonation. Albequerque, New Mexico.

Carroll, M. M. \& Holt, A. C. 1972 Static and dynamic pore-collapse relations for ductile porous materials. J. Appl. Phys. 43 (4), 1626-1636.

Dear, J. P. \& Field, J. E. 1988 A study of the collapse of arrays of cavities. J. Fluid Mech. 190, $409-425$.

Dear, J. P., Field, J. E. \& Walton, A. J. 1988 Gas compression and jet formation in cavities collapsed by a shock wave. Nature 332, 505-508.

Dick, J. J., Hooks, D. E., Menikoff, R. \& Martinez, A. R. 2004 Elastic-plastic wave profiles in cyclotetramethylene tetranitramine crystals. J. Appl. Phys. 96 (1), 374-379. 
Ding, Z. \& Gracewski, S. M. 1996 The behaviour of a gas cavity impacted by a weak or strong shock wave. J. Fluid Mech. 309, 183-209.

Epstein, D. \& Keller, J. B. 1972 Expansion and contraction of planar, cylindrical, and spherical underwater gas bubbles. J. Acoust. Soc. Am. 52 (3), 975.

Ferm, E. N., Morris, C. L., Quintana, J. P., Pazuchanic, P., Stacy, H., Zumbro, J. D., Hogan, G. \& KING, N. 2001 Proton radiography examination of unburned regions in PBX 9502 corner turning experiments. In 12th Am. Phys. Soc. Topical Conf. on Shock Compression of Condensed Matter (ed. M. D. Furnish, N. N. Thadhani \& Y. Horie), pp. 966-969. Georgia.

HaAs, J. F. \& Sturtevant, B. 1987 Interaction of weak shock waves with cylindrical and spherical gas inhomogeneities. J. Fluid Mech. 181, 41-76.

Hu, X. Y. \& KноO, B. C. 2004 An interface interaction method for compressible multifluids. J. Comput. Phys. 198 (1), 35-64.

Jaramillo, E., Sewell, T. D. \& Strachan, A. 2007 Atomic-level view of inelastic deformation in shock loaded molecular crystal. Phys. Rev. B 76, 6.

Johnsen, E. \& Colonius, T. 2009 Numerical simulations of non-spherical bubble collapse. J. Fluid Mech. 629, 231-262.

Johnson, J. N., TANG, P. K. \& Forest, C. A. 1985 Shock-wave initiation of heterogeneous reactive solids. J. Appl. Phys. 57, 4323.

Kang, J., Butler, P. B. \& BAeR, M. R. 1992 A thermochemical analysis of hot spot formation in condensed-phase, energetic materials. Combust. Flame 89, 117-139.

Khasainov, B. A., Borisov, A.A., Ermolaev, B. S. \& Korotkov, A. I. 1981 Two phase visco-plastic model of shock initiation of detonation in high density pressed explosives. In Proc. 7th Symp. (Intl) on Detonation. Annapolis, MN.

Kodama, T. \& Tomita, Y. 2000 Cavitation bubble behaviour and bubble-shock wave interaction near a gelatin surface as a study of in vivo bubble dynamics. J. Appl. Phys. B 70, 139-149.

MADER, C. L. 1965 Initiation of detonation by the interaction of shocks and density discontinuities. Phys. Fluids 8, 1811-1816.

MAIDEN, D. E. \& NutT, G. L. 1986 A hot-spot model for calculating the threshold for shock initiation of pyrotechnic mixtures. In 11th Intl Pyrotechnics Seminar, pp. 813-826. Vail, Colorado.

Menikoff, R. 2001 Compaction wave profiles: simulations of gas gun experiments. J. Appl. Phys. 90 (4), 1754-1760.

Menikoff, R. $2003 a$ Notes on elastic-plastic flow. Tech. Rep. LA-UR-03-0047, Los Alamos National Laboratories, Los Alamos, NM.

Menikoff, R. $2003 b$ Pore collapse and hot spots in HMX. Am. Phys. Soc. Topical Conf., Shock Compression of Condensed Matter (also LA-UR-03-3113), Portand, OR.

Murphy, M. J. \& Adrian, R. J. 2007 Particle response to shock waves in solids: dynamic witness plate/PIV method for detonations. Exper. Fluids 43, 163-171.

Murphy, M. J., Adrian, R. J., Stewart, D. S., Elliott, G. S., Thomas, K. A. \& Kennedy, J. E. 2005 Visualization of blast waves created by exploding bridge wires. J. Visual. 8 (2), 125-135.

Nichols, A. \& TARVER, C. 2002 A statistical hot spot reactive flow model for shock initiation and detonation of solid high explosives. In Proc. 12th Symp. (Intl) on Detonation. San Diego, CA.

Rattray, M. 1951 Perturbation effects in cavitation bubble dynamics. PhD thesis, California Institute of Technology, Pasadena, California.

RAYLEIGH, L. 1917 On the pressure developed in a liquid during the collapse of a spherical cavity. Philos. Mag. 34, 94-98.

Sushchikh, S. Y. \& Nourgaliev, R. R. 2005 Shock waves and flow patterns in shock-induced bubble collapse. 43rd AIAA Aerospace Sciences Meeting and Exhibit (paper no. 2005-1291), Reno, NV.

Tarver, C. M., Chidester, S. K. \& Nichols, A. L 1996 Critical conditions for impact- and shock-induced hot spots in solid explosives. J. Phys. Chem. 100, 5794-5799.

Tarver, C. M., Hallquist, J. O. \& Erickson, L. M. 1985 Modelling short shock pulse duration shock initiation of solid explosives. In Proc. 8th Symp. (Intl) on Detonation. Albuquerque, NM.

Tran, L. \& Udaykumar, H. 2006 Simulation of void collapse in an energetic material. Part 1: Inert case. J. Propul. Power 22 (5), 947-958.

Turangan, C. K., Jamaluddin, A. R., Ball, G. J. \& Leighton, T. G. 2008 Free-Lagrange simulations of the expansion and jetting collapse of air bubbles in water. J. Fluid Mech. 598, 1-25. 